\title{
Technologies for Near-Field Focused Microwave Antennas
}

\author{
Paolo Nepa, Alice Buffi, Andrea Michel, and Giuliano Manara \\ Department of Information Engineering, University of Pisa, Via G. Caruso 16, 56122 Pisa, Italy
}

Correspondence should be addressed to Giuliano Manara; giuliano.manara@iet.unipi.it

Received 7 September 2016; Revised 20 November 2016; Accepted 13 December 2016; Published 19 March 2017

Academic Editor: Shiwen Yang

Copyright (C) 2017 Paolo Nepa et al. This is an open access article distributed under the Creative Commons Attribution License, which permits unrestricted use, distribution, and reproduction in any medium, provided the original work is properly cited.

This paper provides a review spanning different technologies used to implement near-field focused antennas at the microwave frequency band up to a few tens of GHz: arrays of microstrip patches and printed dipoles, arrays of dielectric resonator antennas, reflectarrays, transmitarrays, Fresnel zone plate lenses, leaky-wave antennas, and waveguide arrays.

\section{Introduction}

In the past, near-field focusing techniques based on lens antennas and reflectors have been extensively used at optical and $\mathrm{mm}$-wave bands. Nonetheless, over the last decades, several near-field focused (NFF) antennas have been designed and characterized for applications at lower frequencies. Indeed, NFF antennas are receiving considerable attention in several applications such as RFID (Radio Frequency Identification) systems, gate access control systems, industrial microwave applications, local hyperthermia, and wireless power transfer systems [1].

NFF microwave antennas can be implemented by a number of different technologies and layouts, which can be seen as proper modifications of those that are conventionally used to design and realize far-field (FF) focused antennas. Available solutions include ellipsoidal reflector antennas and pyramidal/conical horns with a dielectric lens in front of the antenna aperture, but they are quite bulky and heavy antennas at the microwave band. Therefore, other technologies that allow for the implementation of planar NFF microwave antennas are preferred. Most of them are array antennas, where the phase of each element current is adjusted to get constructive interference of all field contributions at the focal point.

In this paper, technologies and layouts proposed for the implementation of NFF antennas, at the microwave frequency band up to a few tens of $\mathrm{GHz}$, are revised. The paper is organized as follows. Main parameters and general design criteria for NFF array antennas are concisely introduced in
Section 2, while the reader is referred to $[1,2]$ for a more detailed analysis. Then, a large set of NFF arrays are reviewed in Sections 3-6, which have been classified as follows:

(i) Rectangular (Section 3.1) and circular (Section 3.2) arrays of printed antennas (patches or dipoles)

(ii) Arrays of dielectric resonator antennas (Section 3.3)

(iii) Reflectarrays (Section 4.1)

(iv) Transmitarrays (Section 4.2)

(v) Fresnel zone plate lens antennas (Section 4.3)

(vi) Linear (Section 5.1) and planar (Section 5.2) leakywave antennas

(vii) Waveguide arrays (Section 6)

It is worth noting that the review is limited to those technologies suitable to implement NFF antennas for short-range wireless links at the microwave frequency band (up to a few tens of $\mathrm{GHz}$ ) and does not include the optical devices, such as lenses, dielectrically loaded horns, and reflector mirrors, which are the most valuable technologies at mmwave frequencies and beyond. Moreover, attention has been mostly devoted to NFF antennas for which the antenna size, $L$, and the distance between the focal point and the radiation sources on the array/antenna aperture are both greater than the free-space wavelength $\lambda$. 


\section{Main Features of NFF Array Antennas}

NFF arrays essentially exploit the extreme flexibility of array antennas to control the side lobe level, shape the $-3 \mathrm{~dB}$ focal spot, implement multifocus antennas, and electronically scan the focal point. Important features of the NFF antennas are the focus depth (or depth of focus, DoF), the focus width $W$ at the focal plane, and the level of the secondary lobes around the focal spot region, namely, the axial lobes and the side lobes [1]. Typical parameters of conventional FFfocused antennas (radiation pattern, antenna gain) are also of interest in most of the applications, to quantify the capability of the NFF antenna to minimize the field radiated in the FF region. Indeed, in the context of short-range applications, reducing FF radiation helps to limit the interference with adjacent wireless systems, the effects of unwanted multipath phenomena, and the personnel radiation hazards.

In the conjugate-phase approach [1], the phase of the excitation of each array element is set to compensate for the phase delay introduced by the path between the array element and the assigned focal point, to achieve constructive interference of all the contributions at the focal point. If the focal distance $R_{F}$ is larger enough than the antenna size $L$, then the above phase tapering can be approximated by a quadratic phase profile (Fresnel approximation). Actually, due to the field spreading factor, the peak of the radiated power density does not occur at the focal point where all field contributions sum in phase (focal shift) [1]. The field peak is always located at a point between the antenna aperture and the focal point [1]. Additionally, proper tapering of the amplitude of the excitation may be added to control the level of the secondary lobes around the focal spot region. Indeed, a relatively high level of the secondary lobes around the focal spot may degrade measurement accuracy in noncontact sensing applications, or heat healthy tissues in microwave hyperthermia systems. Also, high secondary lobe level may reduce transmission efficiency in wireless power transfer systems, increase the interference with nearby wireless systems, raise the personnel exposure to radiation hazards, and enlarge the number of false positive readings in RFID systems. Although the conjugate-phase approach is the most used design criteria for NFF antennas, a number of multiobjective optimization techniques have been proposed for reducing the level of the side lobes around the focal spot region, shaping the antenna near field, getting multifocus antennas, or achieving a simultaneous control of the NF radiation and FF pattern [1]. The NFF antennas illustrated in the present review have been designed by using the conjugatephase approach, unless otherwise stated.

In [2], Buffi et al. summarized the basic design criteria for NFF planar square arrays, also giving design and performance curves. The effects of the array geometry on NFF planar array performance have been numerically analyzed in [3] by considering elementary sources and a number of different arrangements for the array elements: ring, ring with cross, four orthogonal arrows, and so forth. There, a quadratic phase profile and a uniform amplitude excitation are assumed for the array excitations. The distance between the elements is kept equal to half a wavelength along the two orthogonal array directions, for any array geometry. The different geometries have been compared in terms of the focal spot size, the side lobe level, and the amplitude of the field at the focal point. This latter has been normalized by dividing the total electric field by the number of array elements, which is between 32 and 52 for the different array arrangements. All the considered arrays occupy an area of $4.5 \lambda \times 4.5 \lambda$, and the focal point is at $5 \lambda$ from the array surface. A similar numerical analysis can be found in [4] for additional array geometries.

As an example, the $-3 \mathrm{~dB}$ and $-6 \mathrm{~dB}$ focused beams of an $8 \times 8 \mathrm{NFF}$ array of $2.4 \mathrm{GHz}$ circularly polarized patches (as those used in [5]) are shown in Figure 1. The interelement spacing is $d=0.8 \lambda$ and the array size is $L \times L=6.4 \lambda \times$ $6.4 \lambda$. The focal distance is $R_{F}=8.2 \lambda \sim 1 \mathrm{~m}$. The phase of the array excitation has been calculated by using the conjugatephase approach and no amplitude tapering is applied. The converging feature of the radiated beam close to focal plane is apparent from the figure, as well as the expected diverging behavior when moving far from the array surface toward the array FF region. The achieved $-3 \mathrm{~dB}$ focus width at the focal plane is $W=14.7 \mathrm{~cm}$, the depth of focus is $\mathrm{DoF}=71 \mathrm{~cm}$, the side lobe level at the focal plane is less than $-15 \mathrm{~dB}$, and the focal shift is $22.5 \mathrm{~cm}$.

\section{NFF Arrays of Microstrip Patches, Printed Dipoles, and Dielectric Resonators}

In this section, planar and circular arrays of printed antennas (patches or dipoles) are reviewed in Sections 3.1 and 3.2, respectively. Section 3.3 is devoted to some implementations of arrays made of dielectric resonators. Finally, a comparison of the performance of some of the above NFF arrays is given in Section 3.4. It is worth noting that printed array technology allows us to realize lightweight and low-profile NFF antennas by easy tuning of the microstrip feeding network with respect to that required by conventional FF-focused arrays.

3.1. NFF Rectangular Arrays of Printed Patches. In this section, a number of NFF rectangular arrays of patch antennas presented in the scientific literature are reviewed, starting from those at the lower frequencies, up to some arrays operating at a few tens of $\mathrm{GHz}$.

A NFF microstrip array for microwave-induced hyperthermia at $433 \mathrm{MHz}$ [6] has been designed by imposing the maximization of the power transfer efficiency [7]. Numerical simulations for the SAR (Specific Absorption Rate) in the array NF region are validated against measurements with an array prototype radiating close to a $20 \mathrm{~cm}$ high glass tank filled with a homogeneous human-body-mimicking material. The tank has the same width as the planar microstrip array, and the measurements are done at $17.4 \mathrm{~cm}$ from the array surface. A $2 \times 4$ planar array $\left(56 \times 50 \mathrm{~cm}^{2}\right.$ wide $)$ of probe-fed rectangular patches has been used for the SAR analysis when a low-loss (conductivity $\sigma=0.04 \mathrm{~S} / \mathrm{m}$ ) and low-permittivity (relative permittivity $\varepsilon_{r}=6.4$ ) fat-mimicking phantom is considered. A SAR analysis in the presence of a high-loss $(\sigma=0.94 \mathrm{~S} / \mathrm{m})$ and high-permittivity $\left(\varepsilon_{r}=57\right)$ bodymimicking phantom has been done using a $3 \times 4$ planar 


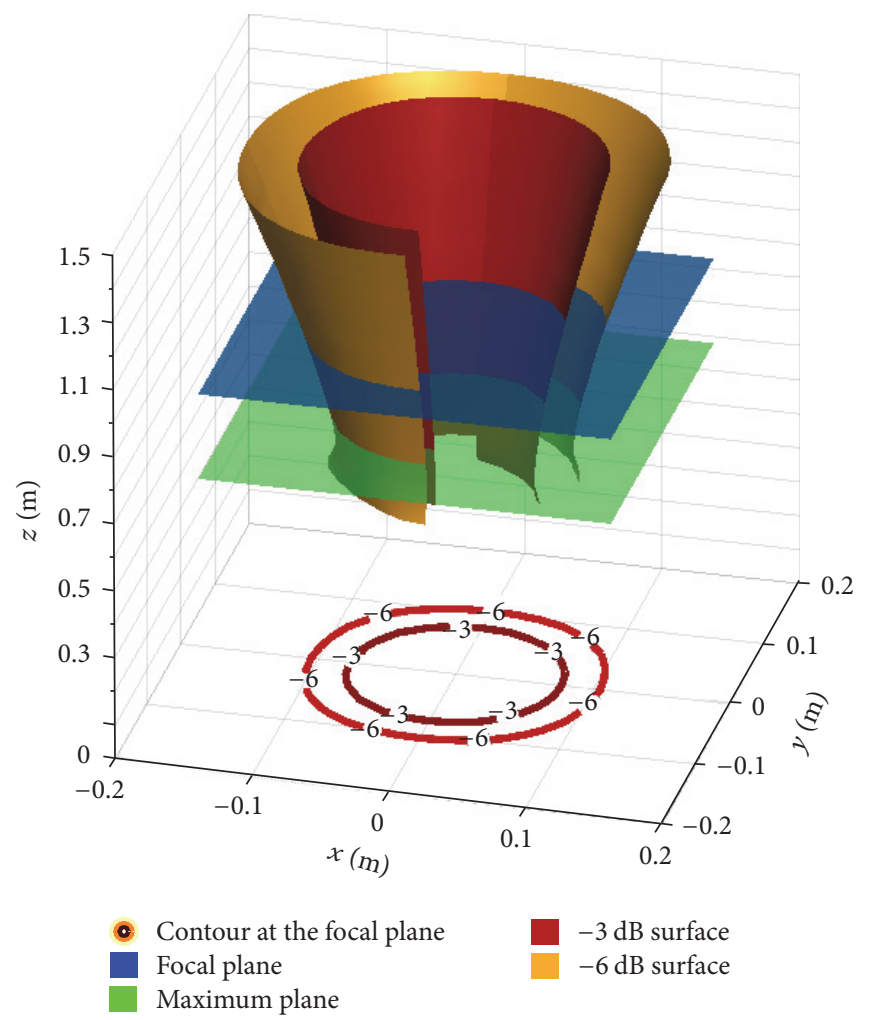

FIGURE 1: Focused beam of the field radiated by an $8 \times 8$ NFF planar array of circularly polarized patches: isosurfaces at $-3 \mathrm{~dB}$ and $-6 \mathrm{~dB}$. The $-3 \mathrm{~dB}$ and $-6 \mathrm{~dB}$ focal spots at the focal plane have been added, in the horizontal plane of the plot. The array is located at the $x y$ surface of the reference system. The focal plane and the maximum field amplitude plane are at around $1 \mathrm{~m}$ and $77.5 \mathrm{~cm}$ from the array surface, respectively.

array of smaller slotted coaxial-fed rectangular patches. In the latter case, the focal point is at a distance of $21 \mathrm{~cm}$ from the array surface; a foam layer and two matching dielectric layers located between the antenna and the body-mimicking phantom are necessary to tune the antenna return loss. For both optimized arrays, the $-3 \mathrm{~dB}$ spot width at the maximumSAR plane is around $8.5 \mathrm{~cm}$.

Two $8 \times 8$ microstrip arrays operating at $1.8 \mathrm{GHz}$ have been proposed for an access control system based on the identification of the mobile phone carried by a person moving through a gate [8]. Both slot-coupled patch arrays are $82 \times$ $82 \mathrm{~cm}^{2}$ large $(5 \lambda \times 5 \lambda)$ and are designed to get a $-3 \mathrm{~dB}$ spot size of $30 \mathrm{~cm}$ at $1.5 \mathrm{~m}$ from the antenna surface. The interelement distance is $0.6 \lambda$. One of the two arrays is linearly polarized with a uniform excitation amplitude. The other one is circularly polarized (CP) and implements $20 \mathrm{~dB}$ Taylor amplitude tapering to satisfy severe constraints on side lobe level performance.

A number of NFF antennas have been proposed for fixed RFID readers at $2.4 \mathrm{GHz}$. Indeed, in those cases where the tagged objects are in the NF region of the reader antenna, a NFF array can allow for reading-rate rising, tag collision mitigation, and item location tracking. Also, in typical RFID portals for supply-chain or warehouse management, adjacent portals are very close to each other and then a NFF reader antenna can limit the interference between nearby portals, as an alternative to expensive and large metallic or absorbing shields. Moreover, a NFF antenna with low side lobe level can reduce the reading errors due to the multipath phenomena. In this context, Buffi et al. [5] designed and prototyped a NFF microstrip array suitable for RFID portals at $2.4 \mathrm{GHz}$. An $8 \times 8$ array of single-feed CP patches has been designed to get a peak of the power density at $1.5 \mathrm{~m}$ from the array surface. Actually, the conjugate-phase tapering has been calculated to focus at $2.7 \mathrm{~m}$ from the array surface, in order to compensate for the focal shift. A sequential rotation technique has been applied to each $4 \times 4$ subarray to improve the axial-ratio performance at the focal spot. Indeed, CP radiation is commonly required for RFID antennas to achieve tag identification independently on the spatial orientation of the tag. It is worth noting that exploiting a subarray structure may reduce the array focusing performance if the focusing conjugate-phase profile applies to each subarray instead of each array element. The interelement distance is $0.8 \lambda$ and whole array size is $80 \times 80 \mathrm{~cm}^{2}(6.4 \lambda \times 6.4 \lambda)$. The NFF array exhibits a $-3 \mathrm{~dB}$ spot size of $20 \mathrm{~cm}$ at $1.5 \mathrm{~m}$ from the array surface, and the depth of focus is around $1.4 \mathrm{~m}$. A further planar microstrip array for $2.4 \mathrm{GHz}$ RFID readers has been designed and characterized in [9]. There, the synthesis technique presented in [10] has been applied to shape the near field of a $13 \times 13$ rectangular array, to achieve an almost constant field amplitude with a minimum ripple in a plane parallel to the array and located at $1 \mathrm{~m}$ from it.

It is apparent that the phased-array concept largely used in radar antennas and smart antennas for mobile communication base stations can also be applied to NFF arrays. Then, 
as an additional feature of a $2.4 \mathrm{GHz}$ RFID system, in [11], a $4 \times 8$ planar array of CP patches has been used to implement a phased NFF array able to move the focal spot in one of the two antenna principal planes, through a set of phase shifters and a microcontroller unit. Comparisons with a conventional RFID reader antenna have been provided in terms of the signal amplitude measured at the reader receiver (RSS, Received Signal Strength).

Again, $2.45 \mathrm{GHz}$ NFF microstrip small arrays of insetfed patches have been designed in $[7,12,13]$ by using an optimization procedure that maximizes the power transfer efficiency between the NFF array and a test antenna located at the assigned focal point. In [7], $4 \times 4$ arrays with a side of around $24 \mathrm{~cm}$ exhibit a spot size less than $9 \mathrm{~cm}$ at focal distances ranging from $9.8 \mathrm{~cm}$ to $19.6 \mathrm{~cm}$. A spot size reduction has been obtained with a bit larger $6 \times 6$ array. Experimental results for another $4 \times 4$ array at $2.45 \mathrm{GHz}$ are given in [13], where the feeding network has been moved to the backside of the microstrip planar array. The same approach as in [7] has been used to design a couple of $4 \times 4$ NFF planar arrays of CP patches operating at $2.4 \mathrm{GHz}$ [14].

The NFF array performance is apparently dependent on the radiation pattern of the specific radiating element that is going to be used. Specifically, increasing the directivity of the array element can be used to raise the power density at the focal spot and reduce the focal spot size and side lobe level, as shown in [15], where a $4 \times 4$ array of patches with a pentagonal shape has been optimized and measured at $2.4 \mathrm{GHz}$. The $40 \times 40 \mathrm{~cm}^{2}$ array in [15] has been compared with a $4 \times 4$ array of conventional rectangular patches [16]. The interelement distance is $0.8 \lambda$ for both arrays. At the focal plane, which is located at $80 \mathrm{~cm}$ from the array surface, the polygonal patch allows reducing the spot size from $17 \times 14 \mathrm{~cm}^{2}$ to $14 \times$ $14 \mathrm{~cm}^{2}$, with a $3 \mathrm{~dB}$ side lobe level reduction too. Moreover, the polygonal patch provides a $2 \mathrm{~dB}$ increase of the power density at the maximum field point, which is located at $30 \mathrm{~cm}$ from the array surface. Since the $4 \times 4$ array of patches in [16] has been designed for hyperthermia treatment, numerical results for the field amplitude in the array axis direction are shown not only in free space but also when the array is located at a distance of $28 \mathrm{~cm}$ in front of a multilayer human-body numerical phantom. More recently, some faceted NFF arrays of microstrip patch antennas at $2.4 \mathrm{GHz}$ have been proposed to increase the power density at the focal point and get a more symmetric focal spot (namely, a value of the ratio $W /$ DoF as close as possible to unity) $[17,18]$.

In the context of X-band applications, two $4 \times 4$ planar microstrip arrays have been designed and tested to be used for noncontact, nondestructive microwave inspection of small material samples in the $10-12 \mathrm{GHz}$ frequency range $[19,20]$. Each $15 \times 15 \mathrm{~cm}^{2}$ wide array is made of linearly polarized coaxial-fed U-slotted patches. The two arrays implement a phase profile to focus at $12.6 \mathrm{~cm}$ and $30 \mathrm{~cm}$ and achieve a $-3 \mathrm{~dB}$ spot size close to $3 \mathrm{~cm}$ and $7.6 \mathrm{~cm}$, respectively. Permittivity measurements on a set of different material samples showed that the NFF array performs as an Xband horn antenna loaded with a dielectric lens, the latter being more bulky and expensive to fabricate. In [21], a radiometric temperature sensor employing a NFF microstrip array was proposed to monitor the temperature of cooked meat products in the food industry, as an alternative to infrared measurements. The NFF $8 \times 8$ array operates at $12.5 \mathrm{GHz}$ and consists of sixteen subarrays. Each subarray is a $2 \times 2$ microstrip array of linearly polarized inset-fed patches. The array is $18.5 \times 18.5 \mathrm{~cm}^{2}$ large $(6.4 \lambda \times 6.4 \lambda$ at $12.5 \mathrm{GHz})$. The $-6 \mathrm{~dB}$ spot width is equal to $6.6 \mathrm{~cm}$, at a distance of $30 \mathrm{~cm}$ from the array plane. $20 \mathrm{~dB}$ Taylor amplitude tapering is used to provide reduced side lobes. Antenna spatial resolution tests have been performed in a hamburger patty-cooking factory, showing that the NFF antenna can resolve individual hamburger patties that are spaced on a square grid with roughly a $12.7 \mathrm{~cm}$ side width.

Dolph-Chebyshev amplitude tapering and a quadratic phase profile have been applied to achieve the desired side lobe level at the focal plane of linearly polarized NFF planar microstrip arrays at $10 \mathrm{GHz}$ [22]. A $4 \times 4$ patch array has been designed and prototyped. The array size is around $8 \times 8 \mathrm{~cm}^{2}$ and the position of the maximum intensity of the field is achieved at $6.2 \mathrm{~cm}$ from the array surface, when a focal length of $20 \mathrm{~cm}$ is assumed to calculate the quadratic phase profile. In addition, numerical results are given for the performance of larger NFF arrays made of $16 \times 16$ and $64 \times 64$ elements, with Dolph-Chebyshev amplitude tapering of $20 \mathrm{~dB}$ and $40 \mathrm{~dB}$, respectively. The focus width in the transverse plane and the focus depth are given for a set of values of the focal length: from $0.5 \mathrm{~m}$ to $2 \mathrm{~m}$ for the $16 \times 16$ array (around $24 \times 24 \mathrm{~cm}^{2}$ wide) and from $3 \mathrm{~m}$ to $11 \mathrm{~m}$ for the $64 \times 64$ array (around $1.4 \times 1.4 \mathrm{~m}^{2}$ wide). The radiation pattern results confirm that the field radiated by a NFF antenna at the focal plane, near the axis of the antenna, has the same properties of the FF radiation pattern of the corresponding FF-focused array [1]. A reduced likeness level appears at the maximum intensity plane, unless the focal shift is small. Small interelement distances may increase mutual coupling between the array elements, so requiring more accurate synthesis techniques. In [22], the effects of the mutual coupling on the NF pattern of $8 \times 8$ Dolph-Chebyshev microstrip arrays have been studied for interelement distances down to half a wavelength.

A $2 \times 8$ NFF planar array of patches operating at $10 \mathrm{GHz}$ has been recently presented in [23], where a subarray structure is proposed to mitigate the mutual coupling between the array elements and reduce the feeding line losses, with respect to arrays using a corporate feeding network. Also, in [24], a NFF sparse planar array of $16 \mathrm{X}$-band patches has been designed by using a genetic algorithm for the optimization of the array element positions, to control the side lobe level.

In some applications, the NFF antenna is required to focus on two or more targets simultaneously (multifocus array). In this context, Álvarez et al. [25, 26] developed an optimization scheme based on the application of the iterative Levenberg-Marquardt optimization algorithm to minimize a least squares problem. In [26], a set of experimental results are shown for a planar array of $8 \times 8$ microstrip inset-fed patches, with an interelement distance of $0.8 \lambda$ at $12 \mathrm{GHz}$. The NFF array is able to realize two simultaneous field amplitude peaks in the array near-field region, which are at $12 \mathrm{~cm}(4.8 \lambda)$ 
and $16 \mathrm{~cm}(6.4 \lambda)$ from the array surface, respectively, out of the array axis. In [27], a $16 \times 16$ NFF planar array consisting of dual-linearly polarized slot-coupled patches has been designed to operate at $12 \mathrm{GHz}$, by implementing a conjugatephase profile. Since the radiating patches are fed through two separated microstrip feeding networks (one for each linear polarization), two simultaneous and independent focused spots can be achieved, one for each linear polarization. The array is $36 \times 36 \mathrm{~cm}^{2}$ wide, and the element spacing is $0.9 \lambda$. Numerical results are shown for two distinct focal points that are both located on a plane at $25 \mathrm{~cm}$ from the array surface. A $-3 \mathrm{~dB}$ spot size of $6.5 \mathrm{~mm}$ has been obtained for the focal point along the array broadside direction.

At higher frequencies, an array of $16 \times 16$ linearly polarized inset-fed microstrip patches has been realized and characterized in [28], where the excitation phases of the array elements are determined by the minimization of a cost function through the steepest descent method. The $17.5 \times$ $15 \mathrm{~cm}^{2}$ wide array is to be used for the remote monitoring of vital signs at $24 \mathrm{GHz}$. The target area is $7.5 \times 7.5 \mathrm{~cm}^{2}\left(36 \lambda^{2}\right)$ wide, and it is located at $12 \mathrm{~cm}$ from the array surface. A focus width of around $7 \mathrm{~cm}$ has been measured at the focal plane.

\subsection{Circular NFF Arrays of Printed Dipoles and Patches. A} circular phased array can be advantageously adopted when the focal spot is required to move along the direction normal to the array surface $[29,30]$. Indeed, only one phase shifter for each array ring is required, as all the array elements on the same ring are equidistant from the focal point. Therefore, $N-1$ phase shifters are needed for a circular array made of $N$ rings. In [29], a prototype of an array of 24 halfwavelength radially oriented printed dipoles has been shown to provide a focus width between 2.8 and $4 \mathrm{~cm}$, when the focal length is varied from 0.3 to $0.9 \mathrm{~m}$ (corresponding to a scan interval of $10 \lambda$ at $5.8 \mathrm{GHz}$ ). The circular array with a tunable focal length has been suggested for RFID systems at $5.8 \mathrm{GHz}$ and is made of three concentric rings, whose radii are 10,30 , and $50 \mathrm{~cm}$, respectively. Only two phase shifters are required. Each ring contains 8 printed dipoles that are uniformly distributed along the ring. A $4 \mathrm{~dB}$ improvement in terms of field amplitude increase at the focal spot region has been obtained in [30], by using a collinear orientation of the dipoles instead of the radial orientation, at the expense of a slightly larger focus depth. However, an optimization of the ring radii with respect to those in [29] is needed to reduce the forelobe and side lobe levels.

A NFF circular array with a fixed focal point on the array axis has been designed in [31]. The radii of two array rings have been calculated with a criterion that resembles that for the design of Fresnel zone plate lens antennas (see Section 4.3):

$$
\sqrt{r_{m}^{2}+R_{F}^{2}}=m \lambda+\sqrt{r_{0}^{2}+R_{F}^{2}}
$$

where $r_{0}$ indicates the radius of the innermost ring, $r_{m}$ denotes the radius of the $m$ th ring, and $R_{F}$ is the focal distance. It is worth noting that in this case all the array elements (and not only those located on the same ring) must be fed in phase to focus at a distance $R_{F}$. This approach simplifies the feeding network implementation but does not help to minimize the far-field radiation, which is usually crucial in most of the NF applications. The prototyped $12 \mathrm{GHz}$ NFF array is made of 16 linearly polarized microstrip patches, uniformly distributed on two rings (4 and 12 elements on the inner and outer ring, resp.). The ring radii are $r_{0}=0.5 \lambda$ and $r_{1}=4.16 \lambda$, and the focal length is set at $R_{F}=7 \lambda$. A further NFF circular array operating at $2.4 \mathrm{GHz}$ has been recently presented in [32]. It consists of 4 and 12 microstrip CP patches on the inner and outer ring, respectively. The array excitations have been determined to maximize the power transfer efficiency between the array and a test antenna (a receiving $\mathrm{CP}$ patch equal to those used for the array) located at the assigned focal point, by following the optimization procedure in [7]. The calculated optimal phases of the array elements sharing the same ring are not equal, as the $\mathrm{CP}$ patches are radially oriented and then a phase delay of $2 \pi /$ (number of elements per ring) must be recovered. All the elements on the same ring could be fed in phase if the CP patches were aligned, as they are in [31]. The inner and outer radii are $5 \mathrm{~cm}$ and $10 \mathrm{~cm}$, respectively, and the maximum intensity point is at $6.5 \mathrm{~cm}$ from the array surface, while the point at which a maximum of the power transmission efficiency was enforced is at $10 \mathrm{~cm}$ from the array surface.

3.3. NFF Planar Arrays of Dielectric Resonators. A set of $8 \times 8$ NFF planar arrays made of CP dielectric resonator antennas have been designed in [33-35] to implement a fixed RFID reader antenna operating at $5.8 \mathrm{GHz}$. Their performance in the NF region is compared against that of the equivalent unfocused array, by numerical simulations. In [34], the array consists of sixteen $2 \times 2$ subarrays. Each subarray consists of four CP single-feed elliptical dielectric resonator antennas and implements a sequential feeding technique to enlarge the antenna axial-ratio bandwidth. The interelement distance is $0.68 \lambda$ and the complete array is $28 \times 28 \mathrm{~cm}^{2}$ large $(5.4 \lambda \times$ $5.4 \lambda$ at $5.8 \mathrm{GHz}$ ). The phase profile has been chosen to focus the radiated field at $40 \mathrm{~cm}(7.37 \lambda)$ from the array surface. A similar numerical analysis has been shown in $[33,35]$, where a dual-feed CP cylindrical dielectric resonator antenna is used for the array elements. Simulated performance at $3.6 \mathrm{GHz}$ of a double-focus $12 \times 12$ planar array of hemispherical dielectric resonator antennas is given in [36], to show the effectiveness of a synthesis procedure that can account for the mutual coupling between the array elements.

Finally, in [37], radiating metallic vias with different diameters have been used as resonators of an X-band NFF $4 \times 4$ array, since the vias are equivalent to open-ended airfilled circular waveguides. Metallic vias are realized into a multilayer substrate made of five $1.5 \mathrm{~mm}$ thick FR4 layers that are bonded together. Since the phase propagation constant depends on the via diameter, each via implements a phase shifter and a radiating element, at the same time. To achieve a compact configuration, an SIW (substrate integrated waveguide) feeding network underneath the layer with the radiating elements is used to excite the vias through coupling slots. The total size of the array is around $10 \times 10 \mathrm{~cm}^{2}$, and it exhibits a peak of the electric field at around $15 \mathrm{~cm}$ from the antenna 


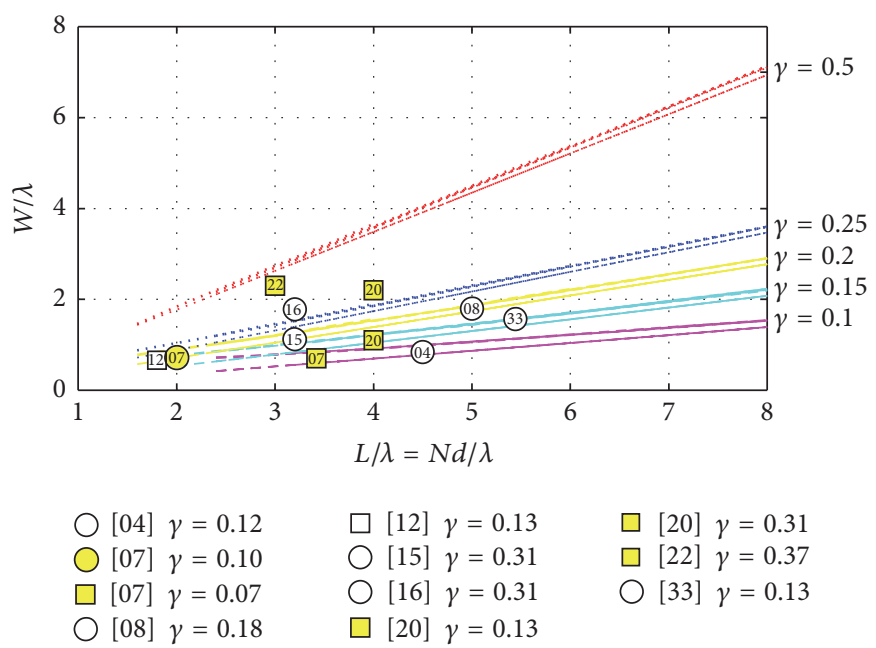

Figure 2: The $-3 \mathrm{~dB}$ spot diameter $W$ (spot size in the transverse plane at the focal distance) as a function of the array size, $L / \lambda=N d / \lambda$, for $0.1<\gamma<0.5$ (see Figure 9 in [2]). The markers denote the performance of NFF arrays described in [4, 7, 8, 12, 15, 16, 20, 22, 33]; the circles and squares are related to simulated and measured results, respectively; for each referenced paper, the empty markers indicate results given by the authors and the filled markers are results extracted from figures. $\gamma$ is the focal distance $R_{F}$ normalized to the radius of the far-field region boundary $\left(2 L^{2} / \lambda\right)$.

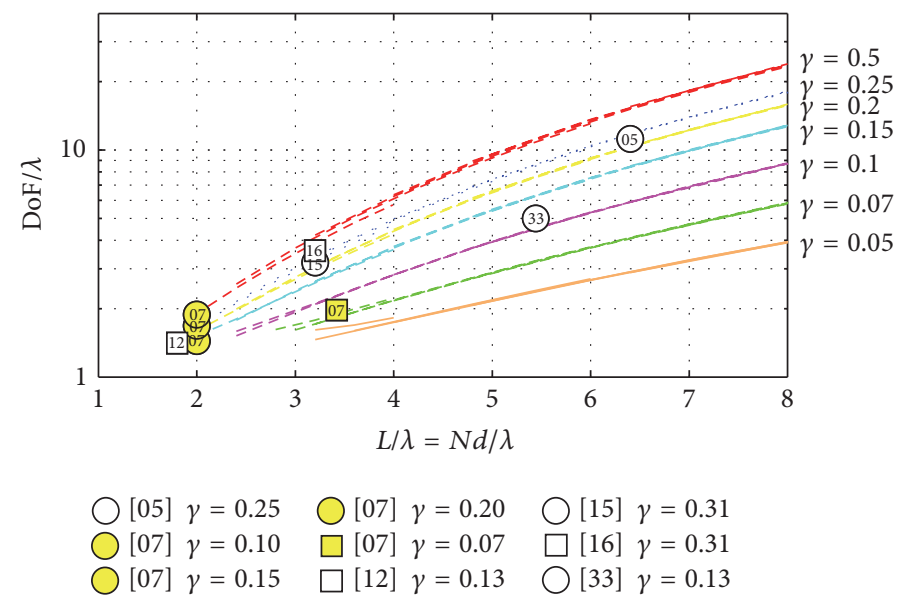

Figure 3: The depth of focus DoF as a function of the array size, $L / \lambda=N d / \lambda$, for $0.05<\gamma<0.5$ (see Figure 10 in [2]). The markers denote the performance of NFF arrays described in $[5,7,12,15,16,33]$; the circles and squares are related to simulated and measured results, respectively; for each referenced paper, the empty markers indicate results given by the authors and the filled markers are results extracted from figures. $\gamma$ is the focal distance $R_{F}$ normalized to the radius of the far-field region boundary $\left(2 L^{2} / \lambda\right)$.

aperture. More recently, a similar structure has been used to realize an $8 \times 8 \mathrm{NFF}$ array at Ka-band $(35 \mathrm{GHz})[38]$.

3.4. Focusing Performance Comparison. To summarize, the focusing performance of some of the above described planar NFF arrays is herein compared with the design curves presented in [2], which have been derived for $N \times N$ squared arrays with interelement distance equal to $d$ along both principal axes. In Figure 2, curves for the $-3 \mathrm{~dB}$ spot diameter, $W$, are shown as a function of the normalized array size, $L / \lambda$, for different values of the normalized focal distance $\gamma=$ $R_{F} /\left(2 L^{2} / \lambda\right)$, in the interval $0.1<\gamma<0.5$. The markers show the performance related to the NFF arrays described in $[4,7,8,12,15,16,20,22,33]$. Besides, curves related to the depth of focus, DoF, are represented in Figure 3, as a function of the array size $L / \lambda$, for $0.05<\gamma<0.5$. The markers represent the performance described in $[5,7,12,15,16,33]$. All results represented by markers in Figures 2 and 3 are also summarized in Table 1.

The numerical results emphasize that, for a given antenna size, both the depth of focus and the focus width increase when the focal point moves far from the array plane. Also, for a given focal distance, focusing performance improves for larger antennas, as predicted by approximate expressions derived for the $-3 \mathrm{~dB}$ focal spot width $W$ of NFF electrically large apertures with a uniform amplitude excitation [1]:

$$
W \sim R_{F}\left(\frac{\lambda}{L}\right)
$$


TABLE 1: $N \times N$ planar focused arrays: performance in terms of depth of focus (DoF) and $-3 \mathrm{~dB}$ spot width $(W)$.

\begin{tabular}{|c|c|c|c|c|c|c|c|}
\hline Ref. & $f_{0}(\mathrm{GHz})$ & $N \times N$ & $L / \lambda$ & $\gamma$ & $W / \lambda$ & $\mathrm{DoF} / \lambda$ & Marker \\
\hline [4] & - & $10 \times 10$ & 4.5 & 0.12 & 0.84 & - & O \\
\hline [5] & 2.4 & $8 \times 8$ & 6.4 & 0.25 & - & 11.2 & O \\
\hline [7] & 2.45 & $4 \times 4$ & 2 & 0.1 & 0.72 & 1.44 & O \\
\hline [7] & 2.45 & $4 \times 4$ & 2 & 0.15 & - & 1.68 & ० \\
\hline [7] & 2.45 & $4 \times 4$ & 2 & 0.2 & - & 1.88 & o \\
\hline [7] & 2.45 & $6 \times 6$ & 3.42 & 0.07 & 0.70 & 1.97 & $\square$ \\
\hline [8] & 1.8 & $8 \times 8$ & 5 & 0.186 & 1.8 & - & 0 \\
\hline [12] & 2.45 & $4 \times 4$ & 1.8 & 0.126 & 0.66 & 1.41 & $\square$ \\
\hline$[15]$ & 2.4 & $4 \times 4$ & 3.2 & 0.31 & 1.12 & 3.2 & O \\
\hline [16] & 2.4 & $4 \times 4$ & 3.2 & 0.31 & 1.78 & 3.6 & O/ם \\
\hline [20] & 10 & $4 \times 4$ & 4 & 0.13 & 1.1 & - & $\square$ \\
\hline [20] & 10 & $4 \times 4$ & 4 & 0.31 & 2.2 & - & $\square$ \\
\hline [22] & 10 & $4 \times 4$ & 3 & 0.37 & 2.3 & - & 口 \\
\hline [33] & 5.8 & $8 \times 8$ & 5.44 & 0.13 & 1.57 & 5 & 0 \\
\hline
\end{tabular}

Equation (2) is directly related to the fact that, in the focal plane, at a distance $R_{F}$, around the spot axis, the NF pattern looks like the FF pattern of the corresponding unfocused antenna [1], and then the angular beamwidth is proportional to $\lambda / L$ (the latter being $0.886 \lambda / L$ and $1.02 \lambda / L$ for rectangular and circular apertures, resp., for a uniform amplitude excitation). However, if $R_{F} / L$ becomes smaller than unity, the spot width $W$ deviates from the above linear behavior and flattens out to a minimum value, which is close to $\lambda / 3$ for focused arrays with a uniform amplitude excitation [39].

\section{NFF Planar Lens Antennas}

Reflectarrays (Figure 4(a)), transmitarrays (Figure 4(b)), and Fresnel zone plate lens (FZPL) antennas (Figure 4(c)) are all considered in this section devoted to NFF planar lens antennas, which can be considered as planar devices able to shape the phase front of the electromagnetic field radiated by the antenna feeder. All of them are suitable to avoid the complexity and losses introduced by the feeding network of electrically large NFF microstrip arrays. On the other hand, spillover can significantly reduce their efficiency. As in conventional FF-focused reflectarrays and transmitarrays, the required phase shift is obtained by modifying one or more geometrical parameters of the unit cell of the quasi-periodic reflecting/transmitting surface.

4.1. NFF Reflectarrays. Reflectarrays combine the promising features of reflector and array antennas into the realization of high-gain FF-focused antennas. In NFF reflectarrays, the required phase of the reflection coefficient at the $n$th element, for focusing at the focal point $F$, can be calculated by using a ray-optics approximation. By considering the geometrical parameters shown in Figure 4(a), it follows that each cell of the quasi-periodic surface has to introduce a phase shift of the reflection coefficient given by

$$
\varphi_{n}=\frac{2 \pi\left(r_{n S}-r_{S}+r_{n F}-r_{F}\right)}{\lambda},
$$

where $r_{n S}$ and $r_{n F}$ are the distances of the $n$th array element from the feeder phase center $S\left(x_{S}, y_{S}, z_{S}\right)$ and the focal point $F\left(x_{F}, y_{F}, z_{F}\right)$, respectively. Moreover, $r_{S}$ and $r_{F}$ are the distances of the reflectarray center from the feeder phase center and the focal point, respectively.

A NFF reflectarray for RFID systems at $2.4 \mathrm{GHz}$ has been designed and characterized in [40]. The circular reflectarray has a diameter of $80 \mathrm{~cm}$ and allows getting a focal spot size of around $20 \mathrm{~cm}$ on a transverse plane at $90 \mathrm{~cm}$ far from the reflectarray center. In [41], the NF performance of the above microstrip reflectarray is compared against that of a planar microstrip [5] array and an ellipsoidal reflector [42]. All above NFF antennas are for $2.4 \mathrm{GHz}$ RFID readers, exhibit the same transversal size $(80 \mathrm{~cm})$, and are designed to get a maximum field amplitude at around $90 \mathrm{~cm}$ from the antenna aperture. The NF radiation characteristics of the offset-fed ellipsoidal reflector antenna have been analyzed through a Physical Optics approximation in [42]; the reflector feed (a CP patch) and the focal point are at the two foci of the ellipsoidal reflector, respectively, which are both in the NF region of the reflector aperture.

A challenging NFF reflectarray design is the one presented by Chou et al. in [43]. The final goal was the design of a dual-band NFF reflectarray suitable for RFID readers at $915 \mathrm{MHz}$ and $2.4 \mathrm{GHz}$, which was required to exhibit a focal spot region at the same position (around $1 \mathrm{~m}$ from the reflectarray surface) at both frequencies. Then, a multilayer double-periodic reflecting surface is needed to implement two different phase profiles, as focusing phase tapering depends on frequency (see (3)). Moreover, since the focal 


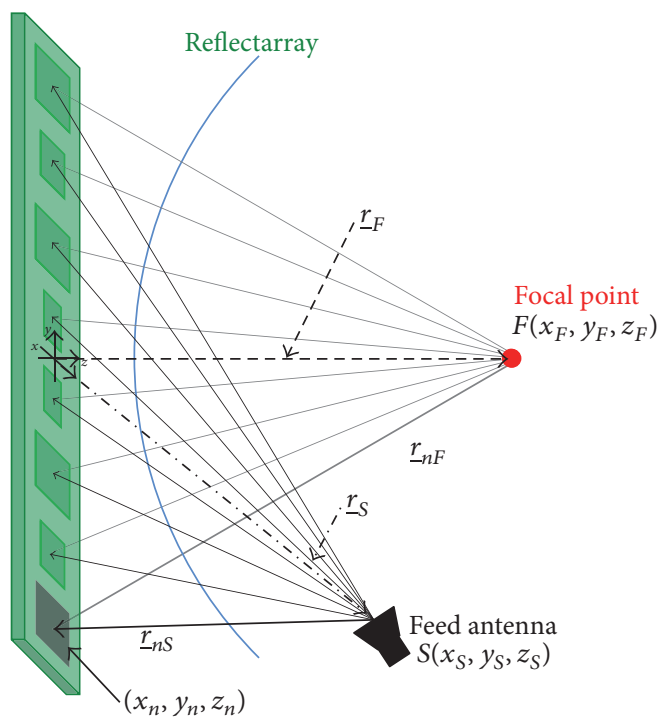

(a)

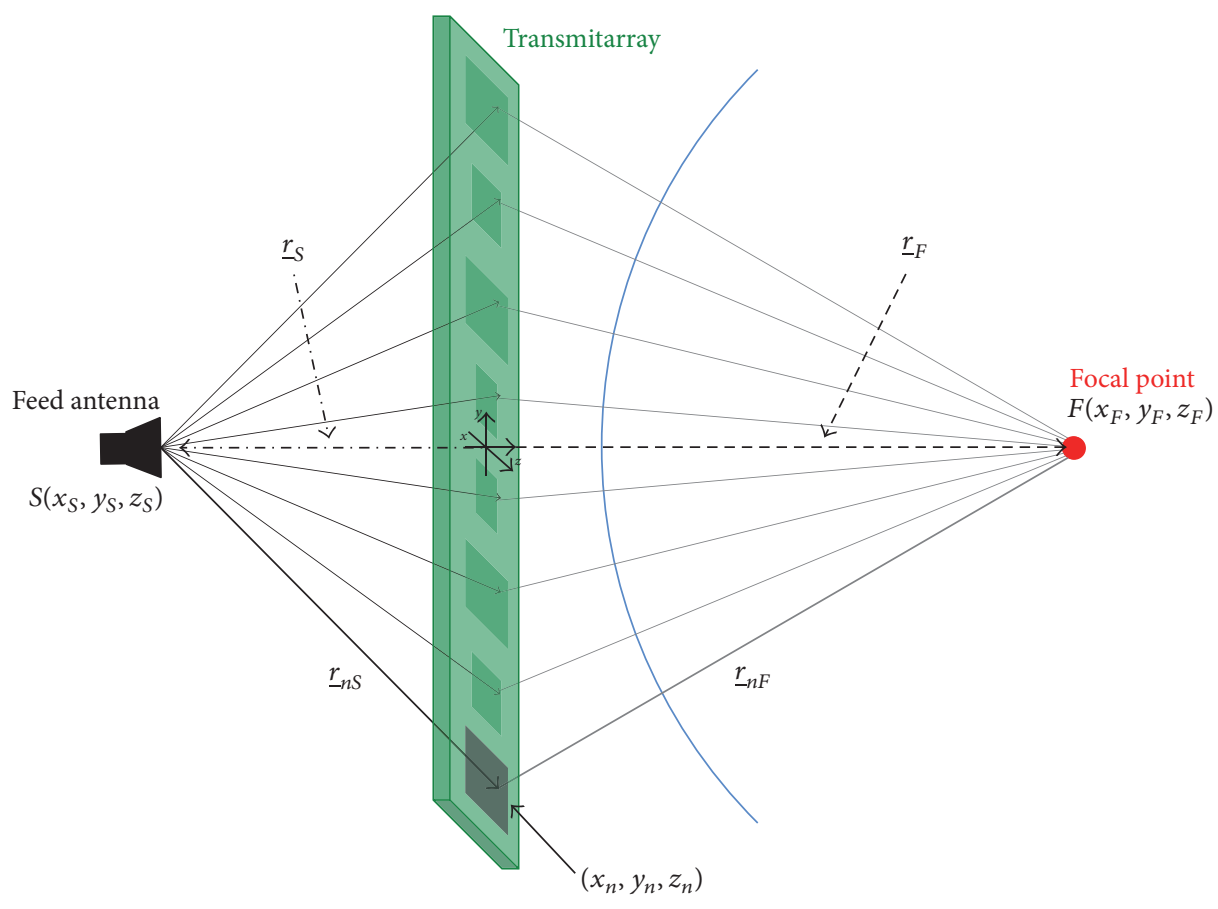

(b)

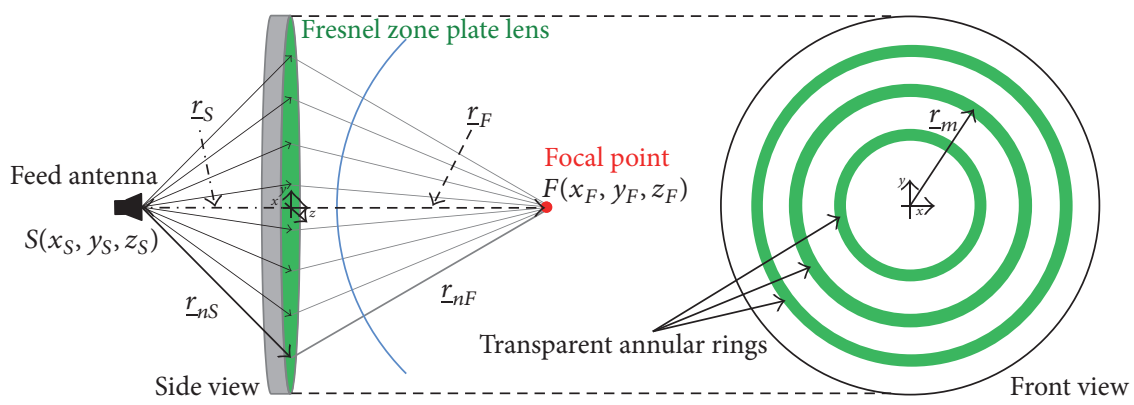

(c)

FIGURE 4: Basic geometrical configurations of different NFF planar lens antennas: (a) reflectarray, (b) transmitarray, and (c) Fresnel zone plate lens. The spherical wave radiated by the feed at $S\left(x_{S}, y_{S}, z_{S}\right)$ interacts with the antenna layer to excite a field that is focused at $F\left(x_{F}, y_{F}, z_{F}\right)$. 
shift depends on frequency, two different focal points must be calculated to synthesize the phase profile. Additionally, for a given size of the dual-band reflectarray, it is apparent that the size of the $-3 \mathrm{~dB}$ focal spot is inherently larger at $915 \mathrm{MHz}$ as the reflecting surface is electrically smaller. At $2.4 \mathrm{GHz}$, the realized prototype shows a peak amplitude at $1.1 \mathrm{~m}$ from the reflectarray, with a focus width of around $32 \mathrm{~cm}$. At the same distance, the focus width goes up to $48 \mathrm{~cm}$ at the lower frequency band. Two CP patches realized in a staked arrangement have been used as the reflectarray feeder, one for each RFID frequency band. As far as the multilayer $80 \times 80 \mathrm{~cm}^{2}$ reflecting surface is concerned, 36 cross-shaped and 144 rectangle-shaped periodicity cells are printed on two different substrates, to implement the required phase profile at $915 \mathrm{MHz}$ and $2.4 \mathrm{GHz}$, respectively. A dual-band reflectarray as that in [43] has been designed and prototyped in [44], by using concentric square rings as reflecting elements at both $915 \mathrm{MHz}$ and $2.4 \mathrm{GHz}$. An optimization procedure based on the steepest descent method (SDM) [28] has been applied in [45] to derive the phase correction required for each reflectarray element, as an alternative to the conventional conjugate-phase profile.

At higher frequencies, a NFF reflectarray operating at $8 \mathrm{GHz}$ has been designed and prototyped to show its effectiveness for H3N2 virus sanitization [46]. The size of the reflectarray is around $16 \mathrm{~cm}$. Both the feed horn and the assigned focal point are located at a distance of $16 \mathrm{~cm}$ from the reflectarray surface. The effectiveness of the NFF reflectarrays has been shown experimentally through a comparison against a conventional slot array. Finally, the NF scanning characteristics of a $\mathrm{Ku}$-band $(14.25 \mathrm{GHz})$ reflectarray have been numerically investigated in [47]. Numerical results are shown for a set of three $36 \mathrm{~cm}$ wide circular reflectarrays, which are focused at a distance of $35.5 \mathrm{~cm}$ from the aperture, when fed by a pyramidal horn antenna. The three subarrays are designed so that the focal point direction is, respectively, at 0,15 , and 30 degrees from the boresight direction in the antenna $E$-plane.

4.2. NFF Transmitarrays. In NFF transmitarrays, the phase compensation to be applied at the $n$th array element for focusing at the focal point $F$ looks like (3) but with the geometrical parameters shown in Figure 4(b). When compared to a reflectarray, a transmitarray does not require an offsetfeed arrangement to avoid the blockage effect, as the feed can be located right in front of the array with no blockage losses. A simple model to analyze a NFF transmitarray is presented in [48], which uses an array-based approach that can account for the spillover effect. However, the proposed approach does not include the field contributions arising from the diffraction phenomena at the transmitarray border. The model has been validated through a comparison with full-wave numerical results, for a set of NFF transmitarrays at $10 \mathrm{GHz}$. An $18 \times 18 \mathrm{NFF}$ transmitarray focused at $6.5 \mathrm{~cm}$ from the antenna aperture exhibits a $1.7 \mathrm{~cm}$ spot size and a side lobe level of around $-13 \mathrm{~dB}$, at the focal plane. If the focal length reduces to $4 \mathrm{~cm}$, then the spot size diminishes to $1.4 \mathrm{~cm}$, although the side lobe level is slightly higher in this case. In [49], a $9 \times 9$ NFF transmitarray using CP dielectric resonator antennas as radiating elements has been designed and compared with the FF-focused version of the same transmitarray. The phase control of the radiating elements is implemented by changing the side length of the square dielectric resonators. The antenna is designed to operate with RFID readers at $5.8 \mathrm{GHz}$. The array size is $21.6 \times 21.6 \mathrm{~cm}^{2}$. The feeding horn and the focal plane are at $28.4 \mathrm{~cm}$ and $40 \mathrm{~cm}$ from the transmitarray surface, respectively. A spot width of $7.8 \mathrm{~cm}$ is obtained at the focal plane. Simulation results for double-focus transmitarrays at $10 \mathrm{GHz}$ have been recently presented in [50]. Each of the $13 \times 13$ unit cells is made of a dielectric square box with four identical holes. The required phase compensation is implemented by changing the radius of the holes, from one cell to another. Firstly, the required phase profile and the corresponding layout of each unit cell have been determined by assuming that the antenna had to focus at a single point, either in the NF region or in the FF region. Later on, a NF/FF double-focus layout is obtained by resorting to a chessboard arrangement of the above designed cells. In this way, the performance of the final layout is in between those of the FF-focused and NF-focused transmitarrays. The array size is $19.5 \times 19.5 \mathrm{~cm}^{2}$. A linearly polarized horn feed and the focal point are positioned at $17.55 \mathrm{~cm}$ and $31 \mathrm{~cm}$ from the array surface, respectively. The simulated $-10 \mathrm{~dB}$ spot region at the focal plane is $6.3 \times 6 \mathrm{~cm}^{2}$ wide. A similar approach has been used to design a doublefocus transmitarray with both focal points in the antenna NF region, which are at a distance from the array surface equal to $31 \mathrm{~cm}$ and $34.7 \mathrm{~cm}$, respectively. The achieved size of the $-10 \mathrm{~dB}$ spot region at the focal plane is $4.8 \times 4.5 \mathrm{~cm}^{2}$ for the focal spot closer to the array and $5.7 \times 5 \mathrm{~cm}^{2}$ for the other one.

It is worth noting that when the theoretical phase compensation in (3) is realized, the focusing performance of a reflectarray/transmitarray is quite similar to that of a planar array with equal size. Small differences are due to the intrinsic amplitude tapering of the equivalent current distribution that is introduced by the spherical spreading factor associated with the feed radiation. The small geometrical differences among the radiating elements of the reflectarray/transmitarray (which are needed to implement the phase profile) usually have a minimum impact with respect to the results that can be obtained by a planar NFF array with identical radiating elements.

4.3. NFF Fresnel Zone Plate Lens Antennas. A Fresnel zone plate lens (FZPL) antenna consists of a distribution of transparent and opaque concentric annuli on a planar surface [51] (Figure 4(c)). The radii of the annuli are calculated such that the field contributions from all transparent annuli add in phase at the targeted focal point $F[52,53]$ :

$$
\left(\sqrt{r_{m}^{2}+r_{S}^{2}}-r_{S}\right)+\left(\sqrt{r_{m}^{2}+r_{F}^{2}}-r_{F}\right)=\frac{m \lambda}{2},
$$

where $r_{m}$ is the radius of the $m$ th ring.

In [53], the $-3 \mathrm{~dB}$ spot size and the side lobe level (at both the targeted focal plane and the maximum field amplitude plane) have been analyzed as a function of the 
focal distance, for a set of FZPL antennas at the Ka-band $(32 \mathrm{GHz})$. Simulations are validated against measurements on two prototypes realized with a $16 \mathrm{~cm}$ diameter circular plate. The focal length for the two antennas is $15 \mathrm{~cm}$ and $45 \mathrm{~cm}$, respectively. The horn feed is at $9.5 \mathrm{~cm}$ from the dielectric plate. For the FZPL antenna with an assigned focal point at $15 \mathrm{~cm}$ from the lens, a $1.24 \mathrm{~cm}$ focus width is obtained at the focal plane. For the prototype with a larger focal length, the focus width goes up to $3.4 \mathrm{~cm}$. Focusing performance degradation is studied when focus scanning is implemented by displacing the horn feed along its transverse axis. As an alternative to a time-consuming mechanical scan, electronic scanning can be implemented by frequency sweeping, as (4) depends on frequency. The focusing performance of a FZPL antenna is studied in [54], when the focal spot is moved along the plate axis by changing the frequency in the $12-18 \mathrm{GHz}$ frequency range ( $\mathrm{Ku}$-band). The diameter of the plate is $44 \mathrm{~cm}$ and the horn feed is at $44 \mathrm{~cm}$ from the plate. At the center frequency of $15 \mathrm{GHz}$, the focal spot is located at $42 \mathrm{~cm}$ from the plate. The focal spot moves in a forward direction from the original position when the frequency is higher than the center frequency, and vice versa. The focus width varies between $2 \mathrm{~cm}$ and $3 \mathrm{~cm}$, in the above frequency range (the higher value corresponding to the upper frequency limit).

A FZPL printed antenna focused in the NF region has been designed and characterized in [55], to be used for freespace material measurements at X-band. The prototype is made of five rings in a circular plate with a diameter less than $42.5 \mathrm{~cm}$. The horn feed and the focal point are both at $30 \mathrm{~cm}$ from the plate. Focusing performance is studied in the 8.2-12 GHz frequency bandwidth, showing a focus width that ranges from $3.4 \mathrm{~cm}$ to $8.5 \mathrm{~cm}$, for different distances from the lens plane (with the smaller focus widths obtained at the upper frequency of the X-band). The radius of the $m$ th ring, $r_{m}$, has been calculated as in [51]

$$
r_{m}=\sqrt{\frac{m \lambda\left(r_{F} r_{S}\right)}{\left(r_{F}+r_{S}\right)}}
$$

which can be derived from (4) if a quadratic approximation is used.

A typical arrangement of focused-beam systems for reliable and nondestructive measurements of the electrical properties of materials in free space consists of two horn antennas and two doubly hyperbolic dielectric lenses. Reid and Smith $[56,57]$ analyzed the performance of such a system when the two doubly hyperbolic lenses are substituted with two grooved-dielectric phase-correcting FZPLs, since they are lighter and thinner than traditional lenses. One side of the FZPL is planar, while the other is properly shaped. In [56], the lens profile has been determined by ray tracing techniques. They are made of Rexolite $\left(\varepsilon_{r}=2.53\right)$ and have a radius of $29.3 \mathrm{~cm}$. They are designed to operate at $10 \mathrm{GHz}$, with a horn feed at $36 \mathrm{~cm}$ from the lens surface. The focal distance is $80.2 \mathrm{~cm}$. It has been verified that the FZPLs and the doubly hyperbolic lenses exhibit similar focusing performance, except that the location of the beam waist for the FZPLs is significantly more frequency-dependent than it is for the doubly hyperbolic dielectric lens. The focal point moves close to the lens if the frequency moves from $10 \mathrm{GHz}$ to $8 \mathrm{GHz}$. Moreover, the power passing through a circle at the focal point with an assigned reference radius is lower for the FZPLs. Most of the power (more than 60\%) passes through a circle with a radius close to $5 \mathrm{~cm}$. In [57], the lens profile has been optimized through a genetic algorithm that has been used to maximize the power passing through a circle of a given radius at the focal plane.

\section{NFF Leaky-Wave Antennas}

Conventional leaky-wave (LW) antennas are based on a travelling wave that radiates continuously along a guiding structure, with a uniform phase constant to produce a FF-focused beam (Figure 5(a)). As far as electrically large antennas are concerned, LW antennas require a simpler feeding mechanism when compared to array antennas, with a reduction of the feeding network losses.

5.1. NFF Linear Leaky-Wave Antennas. A classical approach to focus the radiated field at a point in the antenna NF region consists in a curved guiding structure (Figure 5(b)). In [58], a rectangular waveguide is bent into an equiangular spiral, to focus the field radiated by a uniform and continuous longitudinal slot realized into the narrow wall of the waveguide. All rays emitted by the slot are concentrated at the pole of the spiral (focal point). As regards the $H$-plane power distribution around the focal point, numerical results have been compared with measurements on a prototype operating at $10 \mathrm{GHz}$. An approach that is effective to avoid a bulky curved guiding structure is based on a tapered (or modulated) rectilinear LW guiding structure (Figure 5(c)) [59]. In this case, the complex propagation constant of the leaky mode, $k(x)=\beta(x)-j \alpha(x)$, changes along the rectilinear guiding structure ( $x$-axis), so building up the required phaseconjugated distribution profile on the antenna radiating aperture. By referring to the geometry in Figure 5(c) and resorting to a ray-optics approach, it comes out that the leakymode phase constant has to satisfy the following equation [59]:

$$
\sin \theta_{r}(x)=\frac{\beta(x)}{\beta_{0}}=\frac{\left(x_{F}-x\right)}{\sqrt{\left(x_{F}-x\right)^{2}+z_{F}^{2}}},
$$

where $\theta_{r}(x)$ is the position-dependent radiation angle of the rays emitted by the portion of the LW structure around the abscissa $x$. Also, $\left(x_{F}, 0, z_{F}\right)$ denote the focal point coordinates, and the phase constant is related to the required phase profile on the antenna aperture, $\varphi(x)$, by the relationship $\beta(x)=\beta_{0} \sin \theta_{r}(x)=-d \varphi(x) / d x$. It is worth noting that imposing (6) is equivalent to enforcing the phase condition of a converging cylindrical wave $\varphi(x)=\beta_{0} \sqrt{\left(x_{F}-x\right)^{2}+z_{F}^{2}}=$ $\beta_{0} R(x)$, where $R(x)$ is the distance between the focal point and the point of abscissa $x$ on the antenna aperture. As expected, the latter phase profile agrees with the phaseconjugate profile required on a NFF linear source/array. On the other hand, the leakage rate $\alpha(x)$ controls the tapering 


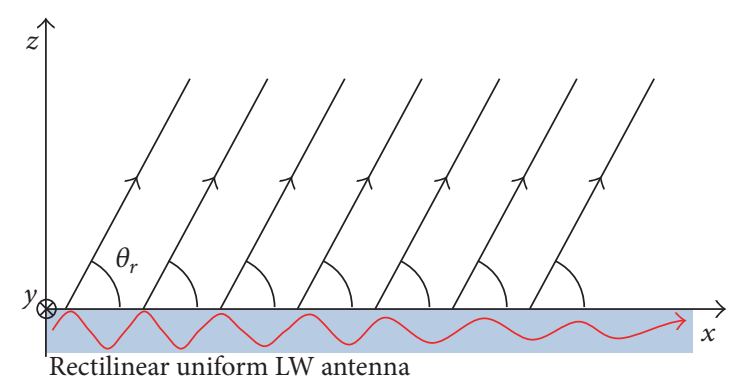

(a)

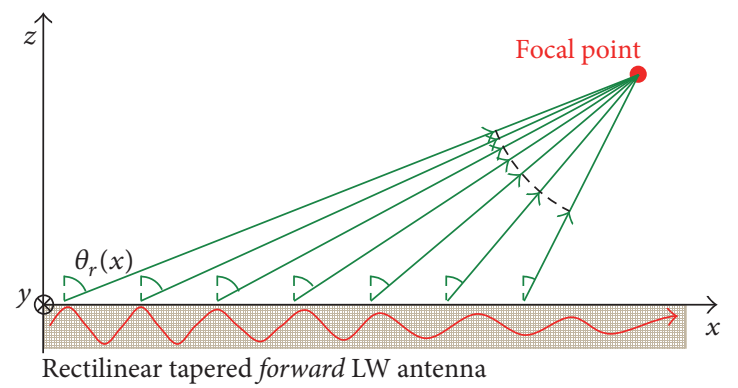

(c)

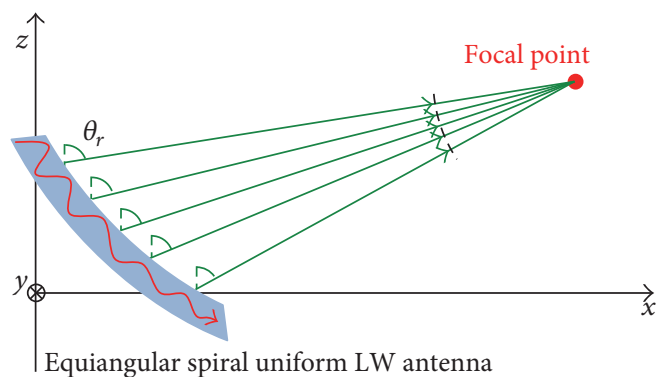

(b)

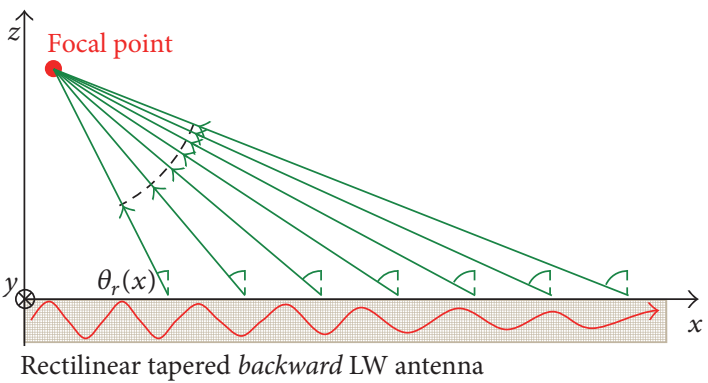

(d)

FiguRE 5: Ray-optics representation of the field radiated by (a) a rectilinear uniform LW antenna focused in the far-field region; (b) a curved (equiangular spiral shaped) uniform LW antenna focused in the near-field region; (c) a rectilinear tapered forward LW antenna focused in the near-field region; (d) a rectilinear tapered backward LW antenna focused in the near-field region. In (a) and (b), the radiation angle $\theta_{r}$ is constant, while in (c) and (d) it varies along the guiding structure.

of the field amplitude on the antenna radiation aperture and then the antenna radiation efficiency.

It is apparent that the most challenging issue when designing a tapered NFF LW antenna is the determination of the geometrical and electrical parameters of the nonhomogeneous rectilinear guiding structure that can allow a simultaneous and (hopefully) independent control of the required phase constant, $\beta(x)$, and leakage rate, $\alpha(x)$, profiles. A rectilinear nonuniform leaky-mode guiding structure can be realized by a waveguide, as, for example, by making the size of the waveguide cross section vary gradually along the longitudinal axis. On the other hand, the planar printed-circuit technology offers low-profile, lightweight, and easily tunable solutions, when compared to waveguide-based LW antennas. A planar nonuniform LW interface, using a metamaterialbased backfire-to-endfire LW antenna, was proposed in [60]. There, the radiated field is calculated through an approximated phased-array approach, and numerical results are shown for two linear NFF LW antennas with a focal length equal to $6 \lambda$ and different lengths of the leaky-mode guiding structure (12 $\lambda$ and $30 \lambda)$.

Gómez-Tornero et al. [61] demonstrated that the hybrid waveguide printed-circuit LW antenna technology (Figure 6) is able to synthesize the simultaneous tapering of the propagation constant and leakage rate, by simply modifying the printed-slot parameters (namely, the slot width and its position along the length of the rectilinear LW antenna). The tapered LW antenna is made of a dielectric-filled rectangular waveguide open on the top where a printed circuit is added in the dielectric-air interface. Two metallic parallel plates are placed along all the printed circuit length to form lateral walls (Figure 6). The slot width $s(x)$ mainly controls the pointing angle $\theta_{r}(x)$ while the slot position $p(x)$ is responsible for the variation of the leakage rate $\alpha(x)$. Simulation results are obtained for a $20 \lambda$-long tapered guiding structure, with a focal distance equal to $25 \lambda$, at $5.5 \mathrm{GHz}$. A further rectilinear nonuniform LW antenna has been analyzed in [62]. There, a width-tapered half-width microstrip LW line embedded between two metallic planes $(8 \mathrm{~mm}$ thick parallelplate waveguide) has been used (Figure 7). A prototype operating at $15 \mathrm{GHz}$ has been implemented with a length of $7.5 \lambda$. Both LW structures in $[61,62]$ are used to realize a one-dimensional NFF LW antenna, namely, 2D focusing in the air space between the two metallic parallel planes (Figure 8). To reduce the spot size and obtain a symmetric (not tilted) NF focal region, in [62], two in-phase LW antennas have been located front-to-front. They are fed at the opposite ends, with separation of one wavelength. The whole antenna length is $32 \mathrm{~cm}(16 \lambda)$. A focus width of $6.7 \mathrm{~mm}$ $(0.33 \lambda)$ and a depth of focus of $25 \mathrm{~mm}(1.25 \lambda)$ have been achieved, with an assigned focal point at $60 \mathrm{~mm}(3 \lambda)$ from the antenna surface. A quite high level of the secondary lobes is observed from both numerical and experimental results. Another one-dimensional NFF LW antenna has been realized by modulating the geometry of a substrate integrated waveguide [63]. The focusing capabilities are shown for a SIW-LW antenna with a length of $15 \mathrm{~cm}(7.5 \lambda$ at $15 \mathrm{GHz})$ and a focal point at $16 \mathrm{~cm}(8 \lambda)$ from the SIW surface.

In case of periodic structures with a period $P$, the Floquet mode propagation constants are given by $\beta_{n}=\beta+2 \pi n / P$, 


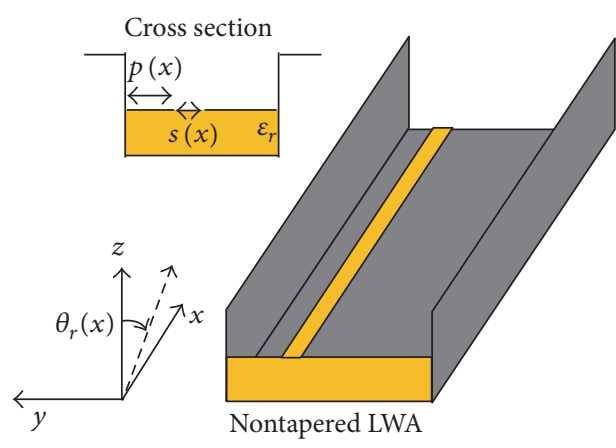

(a)

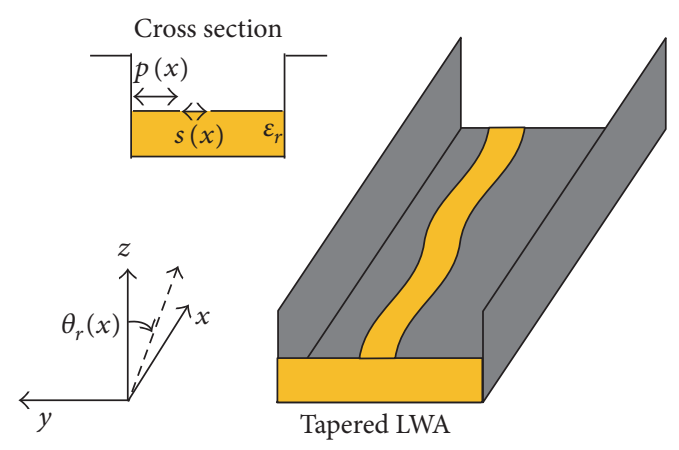

(b)

FIGURE 6: Leaky-wave antenna in hybrid waveguide printed-slot-circuit technology: (a) nontapered LW antenna radiating a far-field focused field; (b) a near-field focused LW antenna based on a tapered printed slot [61].

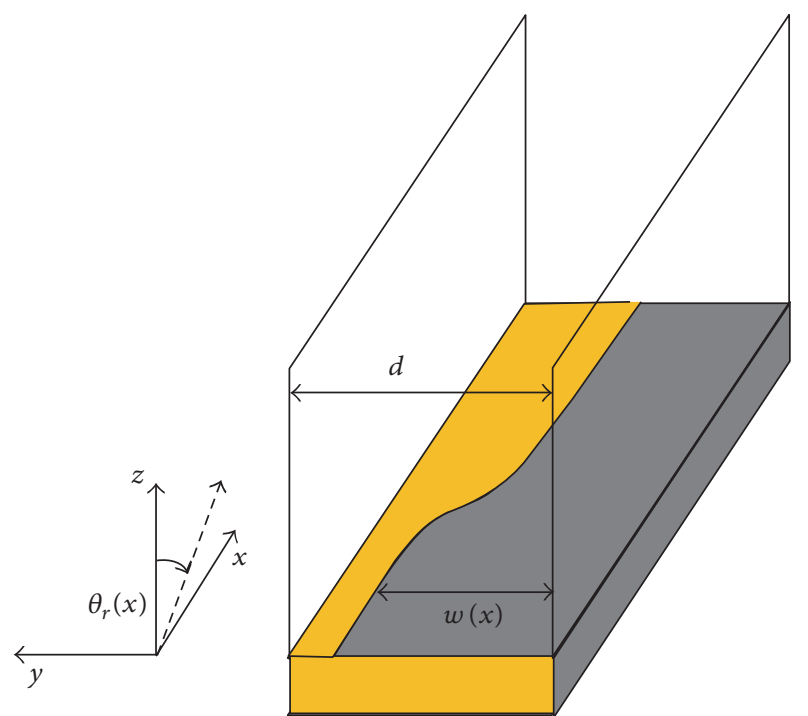

FIGURE 7: A width-tapered half-width microstrip LW line embedded between two metallic planes [63].

where $\beta$ is the propagation constant of the fundamental space harmonic and $n$ is the harmonic order. By a proper choice of the period $P$, it is possible to get a single radiating mode for $n=-1$, such that its corresponding pointing angle is negative:

$$
\sin \theta_{r}=\frac{\beta}{\beta_{0}}-\frac{2 \pi}{\left(P \beta_{0}\right)}<0 .
$$

This means that the LW antenna can radiate a FFfocused backward leaky mode (Figure 5(d)). As for previous nonperiodic structures, tapering of the periodic structure can be used to control the LW pointing angle in (7), to realize a NFF LW antenna. In this context, a nonuniform sinusoidally modulated half-mode microstrip line has been designed to realize a NFF LW antenna at $15 \mathrm{GHz}$ [64]. Both the average value of the strip width and the modulation index of the sine function are used to control the phase constant and the leakage rate of the backward leaky mode, yet avoiding the excitation of spatial harmonics other than that for $n=-1$. Two LW antennas (each one $6 \lambda=120 \mathrm{~mm}$ long) have been assembled front-to-front to get a focal spot at $5.5 \lambda=110 \mathrm{~mm}$ from the radiating aperture. Owing to using a backward LW mode, the feeding point can be advantageously located at the center of the antenna.

A NFF LW antenna can also be obtained by using nonperiodic scatterers (embedded in a scatterer layer) that cause the scattering of the evanescent wave propagating along a waveguide layer, in such a way that the scattered fields sum constructively at the selected focal point $[65,66]$, as illustrated in Figure 9. Constructive interference occurs at the focal point when

$$
\begin{aligned}
& \beta_{g}\left(x_{n}-x_{0}\right)+\beta_{0} \sqrt{\left(x_{F}-x_{n}\right)^{2}+z_{F}^{2}} \\
& -\beta_{0} \sqrt{\left(x_{F}-x_{0}\right)^{2}+z_{F}^{2}}=2 \pi n, \\
& \text { with } n=0,1,2, \ldots,
\end{aligned}
$$

where $\beta_{g}$ is the surface-wave propagation constant, $x_{0}$ is an arbitrary reference point along the waveguide, and $\left(x_{F}, 0, z_{F}\right)$ are the focal point coordinates.

In [66], the surface-wave guiding structure is fabricated by periodically milling rectangular holes on an aluminum plate, which is $40 \mathrm{~cm}$ long and $7.5 \mathrm{~cm}$ wide. Here, the scatterers needed to radiate field contributions at the targeted focal point are obtained by incorporating chirped grating on the waveguide, by intentionally leaving some holes unmilled. A prototype at $20 \mathrm{GHz}$ exhibits a focus width of $9.7 \mathrm{~mm}(0.65 \lambda)$ at $30 \mathrm{~cm}(20 \lambda)$ from the waveguide surface (which is around $26.5 \lambda$ long).

As far as focus-scanned LW antennas are concerned, the inherent dispersion feature of the leaky mode can be used for the synthesis of antennas where the focal point is shifted by changing the signal frequency $[61,62]$. For the NFF LW antenna designed in [61], the properties of the focused beam have been analyzed when changing the frequency in the 4.7-6.5 GHz range. In [62], the focus-scanning properties have been studied in the $14-17 \mathrm{GHz}$ frequency range: the focal length changes from $100 \mathrm{~mm}$ at $14 \mathrm{GHz}$ to $26 \mathrm{~mm}$ at $17 \mathrm{GHz}$ (it results to be $60 \mathrm{~mm}$ at the center frequency of $15 \mathrm{GHz}$ ). A split of the focal spot along the transverse direction is observed at frequencies less than $14.2 \mathrm{GHz}$. If more complex 


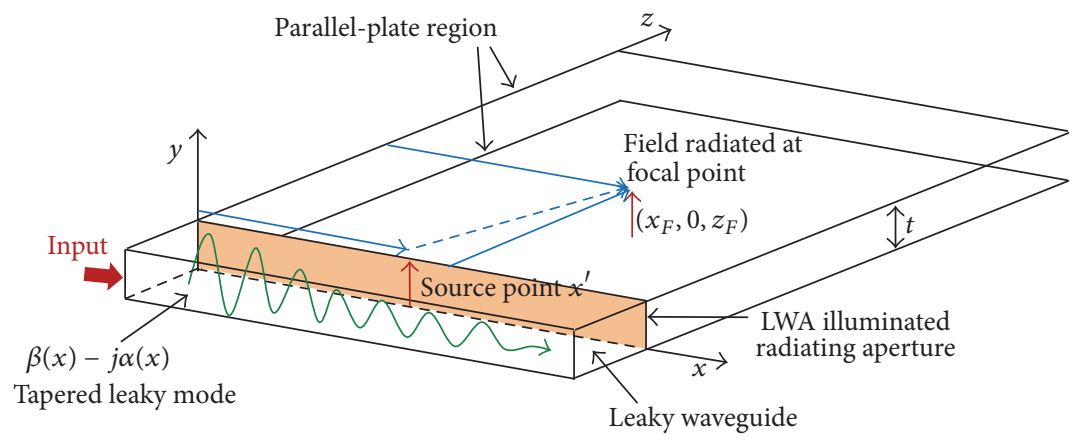

FIGURE 8: A tapered rectilinear leaky-wave antenna embedded between two metallic planes, to implement 2D focusing in the $x z$-plane.

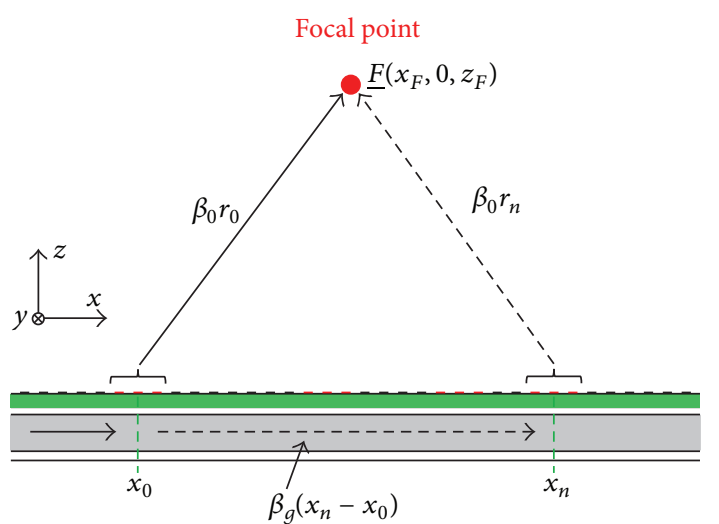

FIGURE 9: A nonuniform periodic scatterer (in red) radiates a portion of the guided wave with a location-dependent phase delay. The scattered waves build up constructive interference at the target point.

shaping of the focal spot is required (namely, not only a focus shift), the LW antenna can be fed through a multitone microwave signal [67]. A set of signal tones can be used to control the focus depth of a single-focus beam, as well as realize a multifocus NFF antenna. In [67], the number of tones and the corresponding frequency and power amplitude have been determined through a minimax optimization procedure. Experimental results are obtained by feeding the antenna developed in [62] with a microwave synthesizer and multitone signals, in the $14-16 \mathrm{GHz}$ frequency interval.

As stated above, in NFF LW antennas, the inherent dispersion feature of the leaky-mode allows for the frequencyscanning of the focal point with no active elements or bulky feeding networks. However, such a solution cannot be applied in those cases where the bandwidth allowed by the standards for the spectrum management is not enough wide to implement the required focus displacement. As an alternative, in [65], the focus scanning is implemented by electronically tuning the pattern of the periodic scatterers on top of the waveguide layer. The scatterers are microstrip line-based fine cascade connections of field effect transistors (FETs). When a group of FETs are switched to the on state, some half-wavelength microstrip strips are formed, which work as resonating scatterers. A prototype operating at $2.4 \mathrm{GHz}$ has been constructed by using 480 FETs, which are uniformly distributed on a parallel-plate waveguide that is $1.5 \mathrm{~m}$ long and $3.2 \mathrm{~cm}$ wide. When the focus is located almost

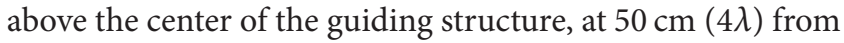
the waveguide surface, the focus width and focal depth are close to $9 \mathrm{~cm}(0.72 \lambda)$ and $26 \mathrm{~cm}(2.1 \lambda)$, respectively. Since the LW antenna is a one-dimensional NFF antenna, the focus width in the direction orthogonal to waveguide and passing through the focal point is quite larger $(60 \mathrm{~cm})$. Finally, it is verified that slight focus scanning (a $7 \mathrm{~cm}$ shift of the focal point) can also be obtained by changing the frequency in a $150 \mathrm{MHz}$ interval around $2.4 \mathrm{GHz}$.

5.2. NFF Planar Leaky-Wave Antennas. The modulated leaky-mode concept developed for linear NFF LW antennas has been extended to planar LW antennas in $[64,68,69]$, to get 3D focusing. In [64], an array of eight $120 \mathrm{~mm}$ long and radially oriented nonuniform sinusoidally modulated halfmode microstrip lines has been implemented at $15 \mathrm{GHz}$, to focus the field at $110 \mathrm{~mm}$ from the array surface. The array is fed at its center and radiates a linearly polarized field. The authors underline the notion that the achieved focus width in the transverse plane (around $15 \mathrm{~mm}$ ) is larger than the one achievable by a NFF circular aperture with the same radius as the array and a uniform aperture field. This is partly due to the fact that the vector field contributions coming at the focal spot from the eight array elements are not aligned (polarization mismatching). The focus width varies between $15 \mathrm{~mm}$ and $20 \mathrm{~mm}$, when considering different longitudinal planes. The measured focal depth is $60 \mathrm{~mm}$. In [68], it has been demonstrated that a cylindrical surface wave can be transformed into a modulated cylindrical backward leaky wave by a quasi-uniform array of double slots. Both the period and the width of the double slots are a function of the surface polar coordinates, to implement the required profiles of the LW phase constant and leakage rate. To validate the concept and synthesis procedure described in [68], a planar printed-circuit prototype, operating at $10 \mathrm{GHz}$ and circularly polarized, has been realized and measured [69]. The final antenna is an Archimedean spiral-shaped doubleprinted slot on the top of a circular dielectric-filled parallelplate waveguide. The antenna geometrical parameters are set to achieve a quadratic phase profile and a quasi-constant amplitude for the field on the radiating aperture. The final 
antenna has a diameter equal to $6.67 \lambda(20 \mathrm{~cm})$. It is fed at its center through a coaxial cable, and the assigned focus is at $4.63 \lambda(13.9 \mathrm{~cm})$ from the antenna surface. A detailed analysis of the copolar and cross-polar components is given, together with a study of the frequency-scanning performance in the 9-11 GHz frequency bandwidth.

\section{NFF Waveguide Arrays}

NFF arrays can also be realized by slotted rectangular waveguides, radial line slot antennas, and arrays of open-ended waveguides. When compared with printed antenna technology, waveguide antennas exhibit a better efficiency and higher power handling. Clauzier et al. [70] designed and characterized a slotted waveguide antenna to be used as the feeder of a transmitarray of an X-band navigation radar, which is $10 \mathrm{~cm}$ wide and $153 \mathrm{~cm}$ long. To this end, I-shaped slots are realized in the narrow side of a rectangular waveguide, to get a 2D focusing effect in the $E$-plane, with a larger beam in the $H$-plane. Using a NFF antenna allows locating the feeder at a distance from the transmitarray as small as $50 \mathrm{~cm}$, so meeting the requirement on the maximum size allowed for the whole radar antenna. The $10 \lambda$-long rectangular waveguide is fed at its center and its two ends are connected to matched loads. The required focusing phase profile along the slotted waveguide (for a focal distance equal to $50 \mathrm{~cm}=15.7 \lambda$ ) has been imposed by an optimization of the slot positions, at $9.41 \mathrm{GHz}$.

Full-wave simulation results for another NFF X-band slotted waveguide array have been presented in [71]. As in [70], the focusing phase profile has been implemented by proper positioning of the radiating slots, even though longitudinal slots are used instead of transverse slots. A set of 10 slotted waveguides have been arranged in a planar star configuration to achieve a $3 \mathrm{D}$ focal spot at the broadside direction. The planar NFF antenna diameter is $36.84 \mathrm{~cm}$ and the focal point is at $10 \mathrm{~cm}$ from the array plane. To avoid a null of the near field at the broadside direction, the radially aligned rectangular waveguides are symmetrically positioned with respect to the antenna E-plane (namely, the left and right half of the planar array are the mirror image of each other). In the context of planar NFF arrays with a circular aperture, similar considerations about the orientation of either linearly polarized radiating elements distributed in the rings of circular arrays or linearly polarized linear antennas arranged in a planar star configuration can also be found in $[29,30,64]$. Indeed, the positioning and orientation of the abovementioned linearly polarized array elements or linear antennas are essential when considering the required polarization and the amplitude maximization of the electric field at the focal point and around it. In this context, it is worth mentioning a NFF radial line slot antenna (RLSA) that has been designed and prototyped in [72], for operations at $12.5 \mathrm{GHz}$. The NFF RLSA has a diameter of $30 \mathrm{~cm}$ and the focal point is at $15 \mathrm{~cm}$ from the antenna aperture. The antenna is fed at its center through a coaxial cable-to-radial waveguide transition. Numerical and experimental results are only shown for the electric field component orthogonal to the array plane, although the latter is apparently a nonradiative field component that rapidly vanishes (with a decrease rate larger than $1 / r$ ) when the focal point moves far from the antenna aperture. Also, the tangential field components exhibit a null at the broadside direction, due to the circular symmetry of the RLSA (the equivalent surface currents are tangential magnetic currents that only depend on the radial distance from the antenna center). The radial field component radiated by a NFF antenna has been considered in very few NFF antenna designs. In this context, Fenn [73] has shown that the radial field component is negligible in a large NFF array of vertical monopoles at $1.3 \mathrm{GHz}$, when the observation point is located at a distance from the array plane equal to one or two aperture diameters. Moreover, Blanco et al. [74] studied a NFF annular-slot leaky-wave antenna at $10 \mathrm{GHz}$, where the goal was to use the normal component to complement the tangential component around the focal spot region, in order to obtain a more symmetric focal spot. Indeed, at the microwave frequency band, the NFF antennas are not so electrically large as those at the mm-wave and optical frequencies, and it follows that the focus depth is always larger than the focus width (the focal spot has an elliptic shape). In [74], the prototyped antenna consists of six concentric-slot pairs and exhibits a $6 \lambda$ diameter at $10 \mathrm{GHz}$, with a focal length equal to $3 \lambda$. Due to the focal shift, the maximum field point is located at $6.5 \mathrm{~cm}=2.17 \lambda$ from the antenna surface. Numerical comparisons are made with results obtained by considering a circular aperture with the same size, when the equivalent surface current is supposed to be aligned along one of the aperture principal axes (not anymore radially oriented). In the latter case, the near field shows a dominant tangential component (parallel to the antenna aperture), while the field normal component is negligible. An increase of the ratio $W /$ DoF up to 0.63 is obtained for the radially polarized annular-slot antenna, thanks to a decrease in the DoF and an increase of the focus width $W(W / \mathrm{DoF}=0.25$ for the ideal aperture distribution used for the comparison). The obtained increase of the $W /$ DoF ratio is due to the fact that all field components are used to estimate the field amplitude around the focal spot: the radial component gives a higher contribution along the antenna axis, while the tangential component is dominant outside the antenna axis. However, the above improvement of the symmetry properties of the focal spot cannot be obtained for any value of the focal length, as the radial field component (nonradiative field component) and the tangential field component (radiative field component) exhibit a different amplitude decay rate in terms of the distance from the antenna surface.

In the context of NFF antennas with a mechanical shift of the focal point, Karnik et al. [75] designed and prototyped a conformal array of open-ended rectangular waveguides, to implement a focused microwave hyperthermia applicator at the K-band $(18-26 \mathrm{GHz})$. An array of waveguides aligned along their longitudinal axis includes a central movable element (named the focusing element) plus a number of surrounding waveguides ( 2 and 4 directing elements, for a linear and a planar array, resp.), which are fixed at their position. The central element can move along its longitudinal axis to both improve the flexibility of the array surface in 
blending with the patient body surface and move the focal spot region around the area to be heated. If the waveguides are fed in phase, a natural focal point is generated at the center of the circle/sphere where the apertures of the waveguides are located. Simulations and measurements confirmed the ability of the array to get focus widths as small as $4 \mathrm{~mm}$, when the focal point moves within a $15 \mathrm{~mm}$ range. Indeed, it is known that a physical curvature of the NFF antenna surface can help to improve the focusing performance [76]. Finally, among the nonplanar NFF arrays, it is worth mentioning the linear array of four Yagi-Uda antennas shown in [77] for wireless power charging of devices at distances of the order of $1 \mathrm{~m}$ from the transmitting antenna. The Yagi antennas are at a distance of 1.8 wavelengths, and they are rotated so that they point toward the desired focal point position to increase the power density around the focal spot.

\section{Conclusions}

Antenna technologies used to implement near-field focused antennas at the microwave frequency band have been reviewed through a comprehensive analysis of the experimental and numerical results presented in the open scientific literature. It has been found that many of the technologies used for conventional unfocused antennas have been revised to implement near-field focused antennas, as, for instance, microstrip arrays, reflectarrays, transmitarrays, Fresnel zone plate lenses, leaky-wave antennas, and waveguide arrays. With respect to the layouts of conventional unfocused antennas, the implementation of the focusing excitation profile for the array currents only requires small layout modifications, as an adjustment of the feeding network in microstrip arrays, or tuning of the geometrical parameters of the quasiperiodic cells in reflectarrays/transmitarrays, or tapering of the guiding structure in leaky-wave antennas. The array excitation profile can be derived by either ad hoc optimization techniques or a simpler phase-conjugate approach.

\section{Competing Interests}

The authors declare that there are no competing interests regarding the publication of this paper.

\section{References}

[1] P. Nepa and A. Buffi, "Near-field focused microwave antennas," IEEE Antennas and Propagation Magazine, vol. 59, 2017.

[2] A. Buffi, P. Nepa, and G. Manara, "Design criteria for nearfield-focused planar arrays," IEEE Antennas and Propagation Magazine, vol. 54, no. 1, pp. 40-50, 2012.

[3] A. M. Abdulkhaleq, K. H. Sayidmarie, R. A. Abd-Alhameed, and E. A. Elkhazmi, "Effects of elements distribution in near focused arrays," in Proceedings of the IEEE 17th International Workshop on Computer Aided Modeling and Design of Communication Links and Networks (CAMAD '12), pp. 343-347, September 2012.

[4] K. H. Sayidmarie and A. M. Abdulkhaleq, "Investigation of six array geometries for focused array hyperthermia applications," Progress In Electromagnetics Research M, vol. 23, pp. 181-194, 2012.
[5] A. Buffi, A. A. Serra, P. Nepa, H. T. Chou, and G. Manara, "A focused planar microstrip array for $2.4 \mathrm{GHz}$ RFID readers," IEEE Transactions on Antennas and Propagation, vol. 58, no. 5, pp. 1536-1544, 2010.

[6] X. He, W. Geyi, and S. Wang, "Optimal design of focused arrays for microwave-induced hyperthermia," IET Microwaves, Antennas and Propagation, vol. 9, no. 14, pp. 1605-1611, 2015.

[7] L. Shan and W. Geyi, "Optimal design of focused antenna arrays," IEEE Transactions on Antennas and Propagation, vol. 62, no. 11, pp. 5565-5571, 2014.

[8] A. Buffi, A. Serra, P. Nepa, G. Manara, and M. Luise, "Near field focused microstrip arrays for gate access control systems," in Proceedings of the IEEE International Symposium on Antennas and Propagation and USNC/URSI National Radio Science Meeting (APSURSI '09), June 2009.

[9] H.-T. Chou, C.-T. Yu, K.-T. Wang, and P. Nepa, "A simple design of patch antenna array with an optimized field distribution in the near-zone for RFID applications," IEEE Antennas and Wireless Propagation Letters, vol. 13, pp. 257-260, 2014.

[10] H.-T. Chou, N.-N. Wang, H.-H. Chou, and J.-H. Qiu, "An effective synthesis of planar array antennas for producing nearfield contoured patterns," IEEE Transactions on Antennas and Propagation, vol. 59, no. 9, pp. 3224-3233, 2011.

[11] H.-T. Chou, M.-Y. Lee, and C.-T. Yu, "Subsystem of phased array antennas with adaptive beam steering in the near-field RFID applications," IEEE Antennas and Wireless Propagation Letters, vol. 14, pp. 1746-1749, 2015.

[12] W. Geyi, “Optimal design of antenna arrays," in Proceedings of the International Workshop on Antenna Technology: Small Antennas, Novel EM Structures and Materials, and Applications (iWAT '14), pp. 143-146, Sydney, Australia, March 2014.

[13] X.-Y. Wang, G.-M. Yang, and W. Geyi, "A new design of focused antenna arrays," Microwave and Optical Technology Letters, vol. 56, no. 10, pp. 2464-2468, 2014.

[14] Y. Jiang, W. Geyi, L. Yang, and H. Sun, "Circularly-polarized focused microstrip antenna arrays," IEEE Antennas and Wireless Propagation Letters, vol. 15, pp. 52-55, 2016.

[15] F. Samadi, "Characteristic improvement of near-field focused array microstrip planar antenna in ISM band," Microwave and Optical Technology Letters, vol. 57, no. 7, pp. 1590-1593, 2015.

[16] F. Tofigh, J. Nourinia, M. Azarmanesh, and K. M. Khazaei, "Near-field focused array microstrip planar antenna for medical applications," IEEE Antennas and Wireless Propagation Letters, vol. 13, pp. 951-954, 2014.

[17] H. Chou and P. Nepa, "Multi-facet focused microwave antennas," in Proceedings of the IEEE-APS Topical Conference on Antennas and Propagation in Wireless Communications (APWC '16), Cairns, Australia, September 2016.

[18] H. Chou and P. Nepa, "Near-field focused radiation by two edge-coupled microstrip antenna arrays," in Proceedings of the URSI International Symposium on Electromagnetic Theory (EMTS '16), pp. 709-712, Espoo, Finland, August 2016.

[19] M. Bogosanovic and A. G. Williamson, "Antenna array with beam focused in near-field zone," Electronics Letters, vol. 39, no. 9, pp. 704-705, 2003.

[20] M. Bogosanović and A. G. Williamson, "Microstrip antenna array with a beam focused in the near-field zone for application in noncontact microwave industrial inspection," IEEE Transactions on Instrumentation and Measurement, vol. 56, no. 6, pp. 2186-2195, 2007. 
[21] K. D. Stephan, J. B. Mead, D. M. Pozar, L. Wang, and J. A. Pearce, "A near field focused microstrip array for a radiometric temperature sensor," IEEE Transactions on Antennas and Propagation, vol. 55, no. 4, pp. 1199-1203, 2007.

[22] S. Karimkashi and A. A. Kishk, "Focused microstrip array antenna using a Dolph-Chebyshev near-field design," IEEE Transactions on Antennas and Propagation, vol. 57, no. 12, pp. 3813-3820, 2009.

[23] P. Li, S. Qu, S. Yang, and Z. Nie, "Sparse focused array antenna based on subarrays," IEEE Antennas and Wireless Propagation Letters, vol. 15, pp. 1-4, 2016.

[24] G. Sun and Q. Zhu, "The design of a focused sparse microstrip antenna array," in Proceedings of the IEEE International Symposium on Antennas and Propagation, pp. 515-516, Fajardo, Puerto Rico, July 2016.

[25] J. Álvarez, R. G. Ayestarán, G. León, J. A. López-Fernández, and F. Las-Heras, "Phase optimization for near field focus on simultaneous targets using antenna arrays," in Proceedings of the 6th European Conference on Antennas and Propagation (EuCAP '12), pp. 2779-2783, IEEE, Prague, Czech Republic, March 2012.

[26] J. A. López-Fernández, J. Álvarez, F. Las-Heras et al., "Near field multifocusing on antenna arrays via non-convex optimisation," IET Microwaves, Antennas \& Propagation, vol. 8, no. 10, pp. 754764, 2014.

[27] J. J. Tomás, M. Arrebola, G. León, and F. Las-Heras, "Near-field focused array with two simultaneous and independent spots," in Proceedings of the IEEE Antennas and Propagation Society International Symposium (APSURSI '12), pp. 1-2, Chicago, Ill, USA, July 2012.

[28] H.-T. Chou, K.-L. Hung, and H.-H. Chou, "Design of periodic antenna arrays with the excitation phases synthesized for optimum near-field patterns via steepest descent method," IEEE Transactions on Antennas and Propagation, vol. 59, no. 11, pp. 4342-4345, 2011.

[29] R. Siragusa, P. Lemaitre-Auger, and S. Tedjini, “Tunable nearfield focused circular phase-array antenna for 5.8-GHz RFID applications," IEEE Antennas and Wireless Propagation Letters, vol. 10, pp. 33-36, 2011.

[30] A. Sharma, I. J. Garcia Zuazola, R. Martinez, J. C. Batchelor, A. Perallos, and L. de-Haro Ariet, "Optimal E-field vector combination for a highly focused antenna-array," IEEE Antennas and Wireless Propagation Letters, vol. 13, pp. 392-395, 2014.

[31] G. Leon and F. L. Heras, "Fresnel-zone-based focused planar array," IEEE Antennas and Wireless Propagation Letters, vol. 13, pp. 165-168, 2014.

[32] Y. Jiang, W. Geyi, and H. Sun, "A new focused antenna array with circular polarization," Microwave and Optical Technology Letters, vol. 57, no. 12, pp. 2936-2939, 2015.

[33] S. H. Zainud-Deen, H. A. Malhat, and K. H. Awadalla, "Dielectric resonator antenna phased array for fixed RFID reader in near field region," in Proceedings of the Japan-Egypt Conference on Electronics, Communications and Computers (JEC-ECC '12), pp. 102-107, Alexandria, Egypt, March 2012.

[34] S. H. Zainud-Deen, H. A. Malhat, and K. H. Awadalla, "Nearfield focusing dielectric resonator antenna array for fixed RFID readers," International Journal of Radio Frequency Identification and Wireless Sensor Networks, vol. 1, no. 2, pp. 1-12, 2011.

[35] S. H. Zainud-Deen, H. A. Maihat, and K. H. Awadalla, " $8 \times 8$ near-field focused circularly polarized cylindrical DRA array for RFID applications," Applied Computational Electromagnetics Society Journal, vol. 27, no. 1, pp. 42-48, 2012.
[36] J. Á. Muñiz, R. G. Ayestarán, J. Laviada, and F. Las-Heras, "Support vector regression for near-field multifocused antenna arrays considering mutual coupling," International Journal of Numerical Modelling: Electronic Networks, Devices and Fields, vol. 29, no. 2, pp. 146-156, 2016.

[37] L. Wang, Y. J. Cheng, and F. Xue, "Design of a near-field-focused substrate integrated planar array antenna," in Proceedings of the 9th European Microwave Integrated Circuits Conference (EuMIC '14)-Held as Part of the 17th European Microwave Week (EuMW '14), pp. 568-571, IEEE, Rome, Italy, October 2014.

[38] Y. J. Cheng and F. Xue, "Ka-band near-field-focused array antenna with variable focal point," IEEE Transactions on Antennas and Propagation, vol. 64, no. 5, pp. 1725-1732, 2016.

[39] R. C. Hansen, "Minimum spot size of focused apertures," in Electromagnetic Wave Theory, Part 2, pp. 661-668, Pergamon Press, 1965.

[40] H.-T. Chou, T.-M. Hung, N.-N. Wang, H.-H. Chou, C. Tung, and P. Nepa, "Design of a near-field focused reflectarray antenna for $2.4 \mathrm{GHz}$ RFID reader applications," IEEE Transactions on Antennas and Propagation, vol. 59, no. 3, pp. 1013-1018, 2011.

[41] H.-T. Chou, T.-M. Hung, N.-N. Wang, H.-H. Chou, and P. Nepa, "Characteristic comparisons between array, reflectarray and elliptic reflector antennas in RFID near-field focused communications," in Proceedings of the IEEE International Conference on Wireless Information Technology and Systems (ICWITS '10), pp. 1-4, IEEE, Honolulu, Hawaii, USA, September 2010.

[42] H.-T. Chou, L.-R. Kuo, H.-H. Chou, K.-L. Hung, and P. Nepa, "Realistic implementation of ellipsoidal reflector antennas to produce near-field focused patterns," Radio Science, vol. 46, no. 5, Article ID RS5007, 2011.

[43] H.-T. Chou, P.-H. Hsueh, T.-M. Hung, L.-R. Kuo, and H.-H. Chou, "A dual-band near-field focused reflectarray antenna for RFID applications at 0.9 and $2.4 \mathrm{GHz}$," Radio Science, vol. 46, no. 6, Article ID RS6010, 2011.

[44] B.-Q. You, Y.-X. Liu, J.-H. Zhou, and H.-T. Chou, "Numerical synthesis of dual-band reflectarray antenna for optimum nearfield radiation," IEEE Antennas and Wireless Propagation Letters, vol. 11, pp. 760-762, 2012.

[45] H.-T. Chou, Y.-X. Liu, X.-Y. Dong, B.-Q. You, and L.-R. Kuo, "Design of reflectarray antennas to achieve an optimum nearfield radiation for RFID applications via the implementation of SDM procedure," Radio Science, vol. 50, no. 4, pp. 283-293, 2015.

[46] W.-T. Hung, J.-J. Tung, and S.-Y. Chen, "A focusing reflectarray and its application in microwave virus sanitizer," Radio Science, vol. 49, no. 10, pp. 890-898, 2014.

[47] P. Nayeri, A. Z. Elsherbeni, R. L. Haupt, and F. Yang, "Near-field scanning characteristics of focused reflectarray antennas," in Proceedings of the 31st International Review of Progress in Applied Computational Electromagnetics (ACES '15), Williamsburg, Va, USA, March 2015.

[48] E. G. Plaza, G. León, S. Loredo, and F. Las-Heras, "Near-field focusing transmitarray lens," in Proceedings of the 9th European Conference on Antennas and Propagation (EuCAP '15), 5, 1 pages, Lisbon, Portugal, April 2015.

[49] S. H. Zainud-Deen, S. M. Gaber, H. A. Malhat, and K. H. Awadalla, "Multilayer dielectric resonator antenna transmitarray for near-field and far-field fixed rfid reader," Progress in Electromagnetics Research: C, vol. 27, pp. 129-142, 2012.

[50] H. A. Malhat, W. M. Hassan, and S. H. Zainud-Deen, "Different designs of dual-focus perforated transmitarray antenna in near/far-field region," Advanced Electromagnetics, vol. 4, no. 3, pp. 25-34, 2015. 
[51] H. Hristov, Fresnel Zones in Wireless Links, Zone Plate Lenses and Antennas, Artech House, Norwood, Mass, USA, 2000.

[52] S. Karimkashi and A. A. Kishk, "A new fresnel zone antenna with beam focused in the fresnel region," in Proceedings of the 29th General Assembly of the International Union of Radio Science, pp. 1-4, Chicago, Ill, USA, August 2008.

[53] S. Karimkashi and A. A. Kishk, "Focusing properties of fresnel zone plate lens antennas in the near-field region," IEEE Transactions on Antennas and Propagation, vol. 59, no. 5, pp. 1481-1487, 2011.

[54] Z. Li, Z. Wang, J. Wu, and H. Tian, "Axially steered spots of nearfield fresnel zone plate lens with sweeping frequency," IEEE Antennas and Wireless Propagation Letters, vol. 13, pp. 955-958, 2014.

[55] M. B. Perotoni and C. Junqueira, "X-band printed fresnel zone plate antenna: design, simulation, and field measurements," Microwave and Optical Technology Letters, vol. 57, no. 11, pp. 2604-2609, 2015.

[56] D. R. Reid and G. S. Smith, "A comparison of the focusing properties of a Fresnel zone plate with a doubly-hyperbolic lens for application in a free-space, focused-beam measurement system," IEEE Transactions on Antennas and Propagation, vol. 57, no. 2, pp. 499-507, 2009.

[57] D. R. Reid and G. S. Smith, "The optimization of Fresnel zone plates for use in focused-beam measurement systems," in Proceedings of the IEEE Antennas and Propagation Society International Symposium (APSURSI '09), pp. 1-4, North Charleston, SC, USA, June 2009.

[58] I. Ohtera, "Focusing properties of a microwave radiator utilizing a slotted rectangular waveguide," IEEE Transactions on Antennas and Propagation, vol. 38, no. 1, pp. 121-124, 1990.

[59] P. Burghignoli, F. Frezza, A. Galli, and G. Schettini, "Synthesis of broad-beam patterns through leaky-wave antennas with rectilinear geometry," IEEE Antennas and Wireless Propagation Letters, vol. 2, no. 1, pp. 136-139, 2003.

[60] I.-H. Lin, C. Caloz, and T. Itoh, "Near-field focusing by a nonuniform leaky-wave interface," Microwave and Optical Technology Letters, vol. 44, no. 5, pp. 416-418, 2005.

[61] J. L. Gómez-Tornero, F. Quesada-Pereira, A. Alvarez-Melcón, G. Goussetis, A. R. Weily, and Y. J. Guo, "Frequency steerable two dimensional focusing using rectilinear leaky-wave lenses," IEEE Transactions on Antennas and Propagation, vol. 59, no. 2, pp. 407-415, 2011.

[62] A. J. Martínez-Ros, J. L. Gómez-Tornero, F. J. ClementeFernandez, and J. Monzó-Cabrera, "Microwave near-field focusing properties of width-tapered microstrip leaky-wave antenna," IEEE Transactions on Antennas and Propagation, vol. 61, no. 6, pp. 2981-2990, 2013.

[63] A. J. Martínez-Ros, J. L. Gómez-Tornero, and G. Goussetis, "Holographic pattern synthesis with modulated substrate integrated waveguide line-source leaky-wave antennas," IEEE Transactions on Antennas and Propagation, vol. 61, no. 7, pp. 3466-3474, 2013.

[64] A. J. Martinez-Ros, J. L. Gómez-Tornero, V. Losada, F. Mesa, and F. Medina, "Non-uniform sinusoidally modulated half-mode leaky-wave lines for near-field focusing pattern synthesis," Institute of Electrical and Electronics Engineers. Transactions on Antennas and Propagation, vol. 63, no. 3, pp. 1022-1031, 2015.

[65] Y. Monnai and H. Shinoda, "Focus-scanning leaky-wave antenna with electronically pattern-tunable scatterers," IEEE Transactions on Antennas and Propagation, vol. 59, no. 6, pp. 2070-2077, 2011.
[66] T. Okuyama, Y. Monnai, and H. Shinoda, "20-ghz focusing antennas based on corrugated waveguide scattering," IEEE Antennas and Wireless Propagation Letters, vol. 12, pp. 12841286, 2013.

[67] J. L. Gómez-Tornero, A. J. Martínez-Ros, and J. Monzó-Cabrera, "Demonstration of simple electronic near-field beamforming using multitone microwave signals with a leaky-wave focused applicator," IEEE Antennas and Wireless Propagation Letters, vol. 14, pp. 143-146, 2015.

[68] J. L. Gomez-Tornero, D. Blanco, E. Rajo-Iglesias, and N. Llombart, "Holographic surface leaky-wave lenses with circularlypolarized focused near-fields-part I: concept, design and analysis theory," IEEE Transactions on Antennas and Propagation, vol. 61, no. 7, pp. 3475-3485, 2013.

[69] D. Blanco, J. L. Gómez-Tornero, E. Rajo-Iglesias, and N. Llombart, "Holographic surface leaky-wave lenses with circularly-polarized focused near-fields-part II: experiments and description of frequency steering of focal length," IEEE Transactions on Antennas and Propagation, vol. 61, no. 7, pp. 3486-3494, 2013.

[70] S. Clauzier, S. Avrillon, L. Le Coq, M. Himdi, F. Colombel, and E. Rochefort, "Slotted waveguide antenna with a near-field focused beam in one plane," IET Microwaves, Antennas and Propagation, vol. 9, no. 7, pp. 634-639, 2015.

[71] M. L. Chen, S. Gupta, Z. L. Ma, and L. J. Jiang, "Linearly polarized near field focused slot-array waveguide," in Proceedings of the IEEE Antennas and Propagation Society, AP-S International Symposium (APSURSI '14), pp. 1047-1048, Memphis, Tenn, USA, July 2014.

[72] M. Ettorre, M. Casaletti, G. Valerio et al., "On the near-field shaping and focusing capability of a radial line slot array," IEEE Transactions on Antennas and Propagation, vol. 62, no. 4, pp. 1991-1999, 2014.

[73] A. J. Fenn, "On the radial component of the electric field for a monopole phased array antenna focused in the near zone," IEEE Transactions on Antennas and Propagation, vol. 40, no. 6, pp. 723-727, 1992.

[74] D. Blanco, J. L. Gomez-Tornero, E. Rajo-Iglesias, and N. Llombart, "Radially polarized annular-slot leaky-wave antenna for three-dimensional near-field microwave focusing," IEEE Antennas and Wireless Propagation Letters, vol. 13, pp. 583-586, 2014.

[75] N. S. Karnik, R. Tulpule, M. Shah et al., "Design, simulation and experimental study of near-field beam forming techniques using conformal waveguide arrays," IET Microwaves, Antennas \& Propagation, vol. 4, no. 2, pp. 162-174, 2010.

[76] R. W. Bickmore, "On focusing electromagnetic radiators," Canadian Journal of Physics, vol. 35, no. 11, pp. 1292-1298, 1957.

[77] R. Van der Linden and H. J. Visser, "Analysis, design and realization of a near-field focused RF power transfer system," Journal of Physics: Conference Series, vol. 476, no. 1, Article ID 012118, pp. 1-5, 2013. 


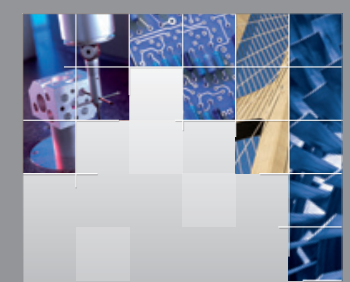

\section{Enfincering}
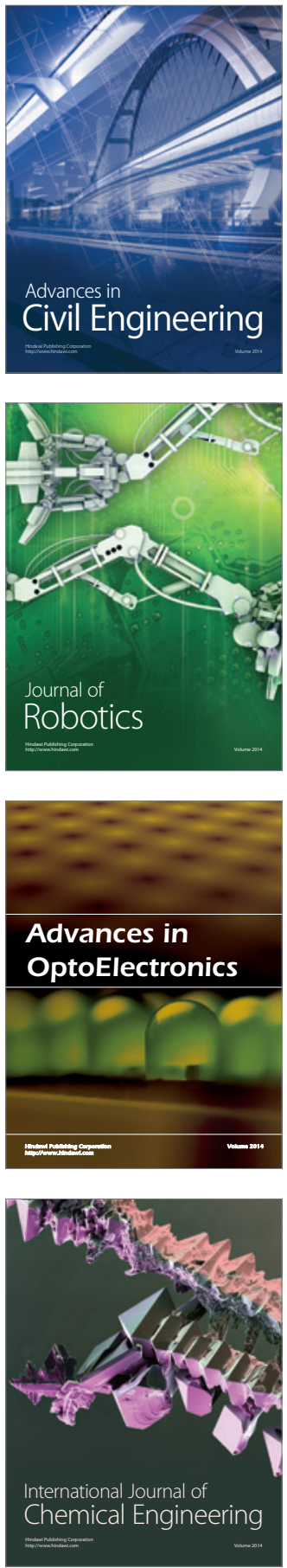

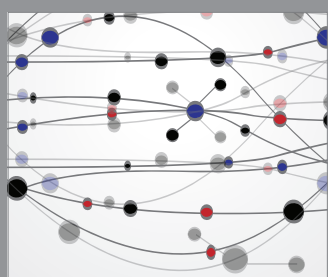

The Scientific World Journal

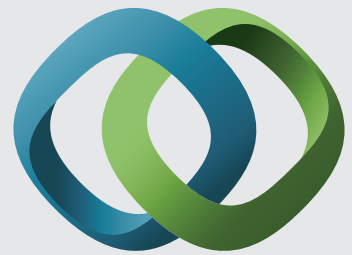

\section{Hindawi}

Submit your manuscripts at

https://www.hindawi.com
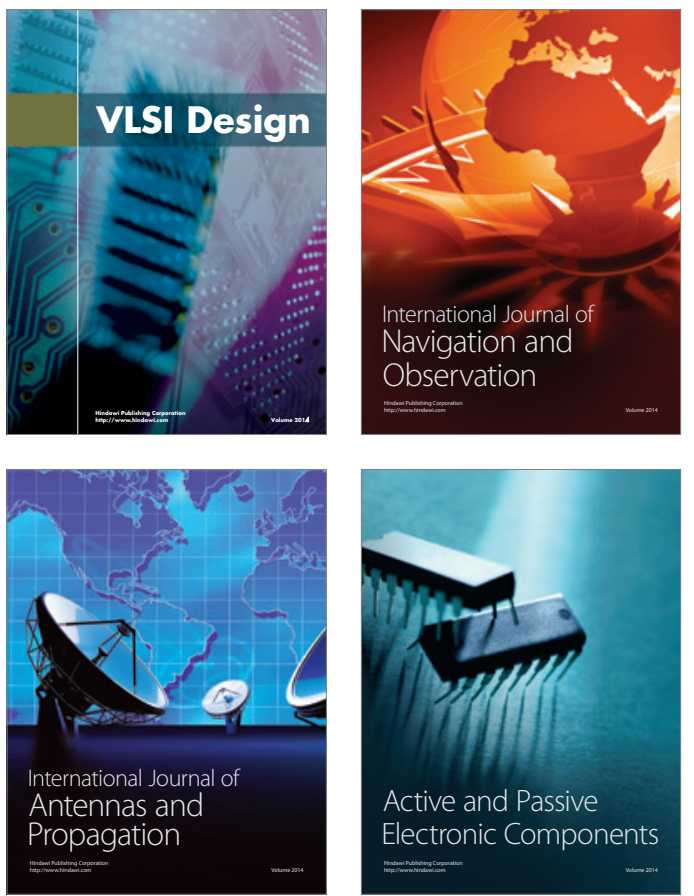
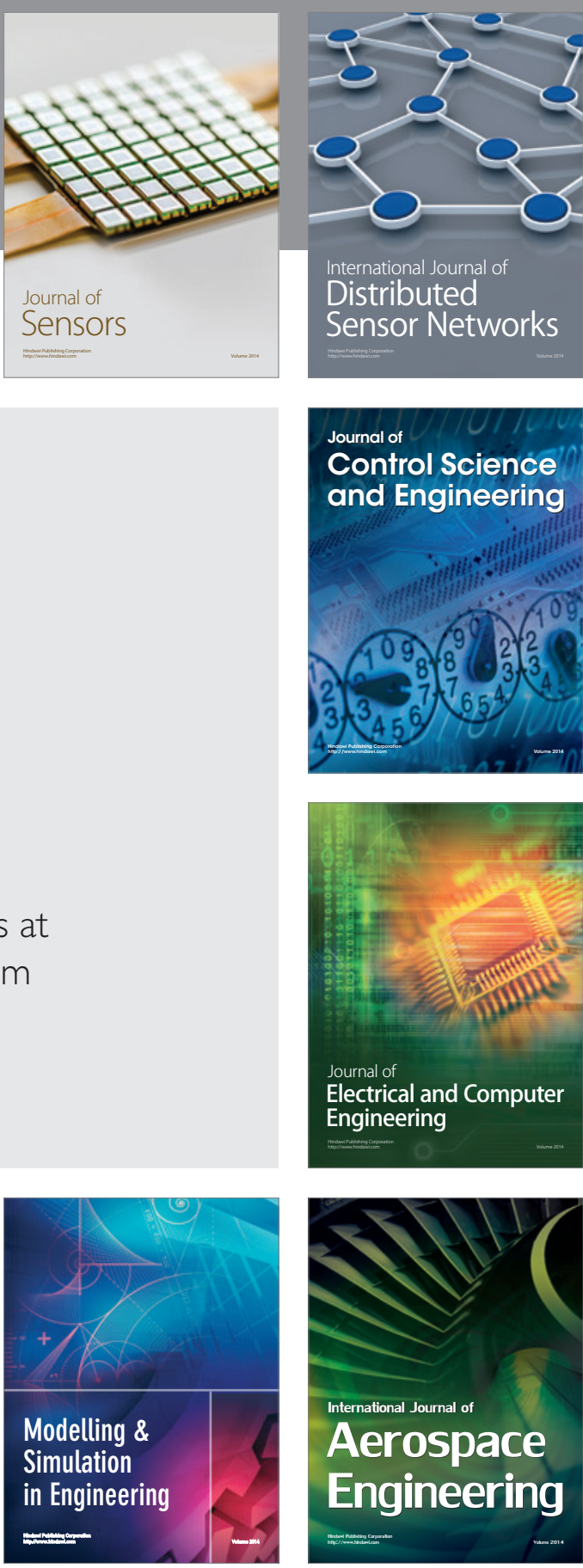

International Journal of

Distributed

Sensor Networks

$-$

Joumal of

Control Science

and Engineering
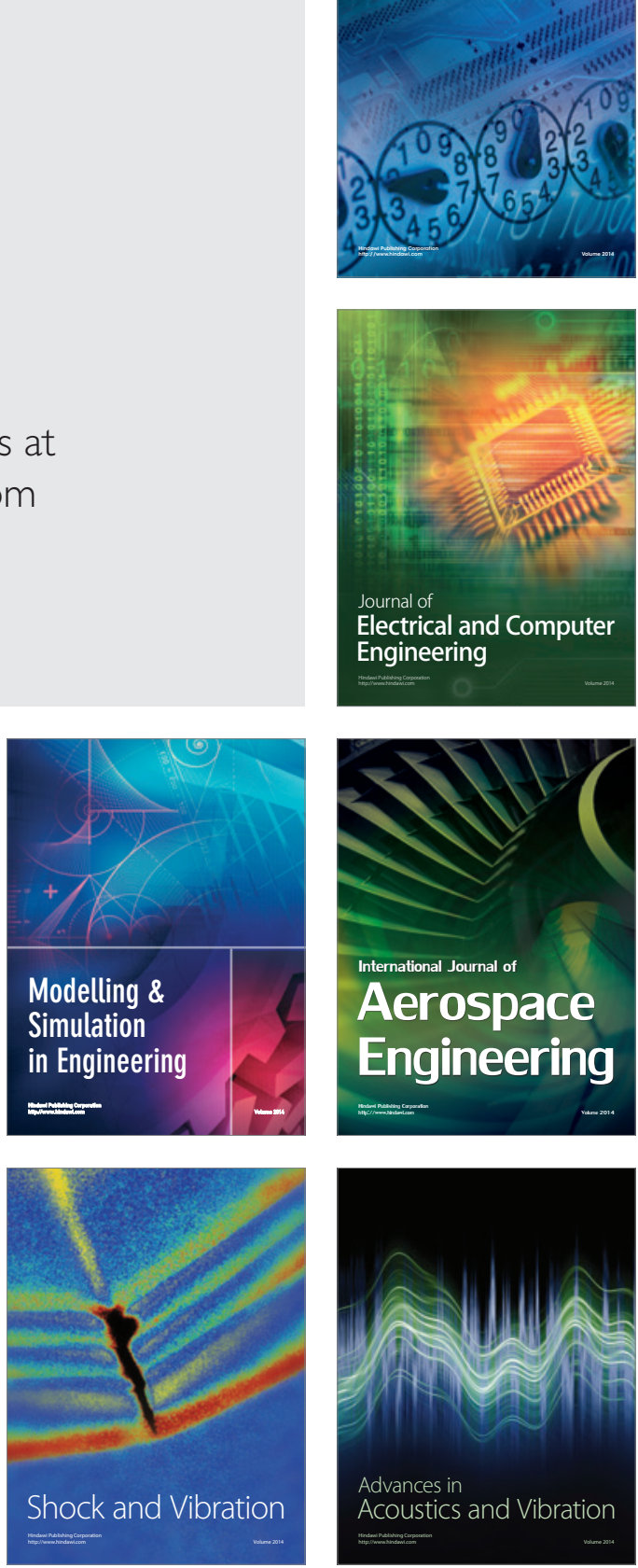